\title{
Research on Structural Flexibility and Acceptance Model (SFAM) Reconstruction based on Disruption Innovation in the Social Humanities and Education Sector
}

\author{
SOLIMUN, ADJI ACHMAD RINALDO FERNANDES, INTAN RAHMAWATI, \\ LAILIL MUFLIKHAH, FARID UBAIDILLAH, ALIFYA AL ROHIMI, \\ Department of StatisticsBrawijaya University \\ Veteran Street, Malang City, East Java \\ INDONESIA
}

\begin{abstract}
The research objectives are as follows: (1) Developing a flexible structural model of the relationship between variables. (2) Develop a structural model that is robust with the assumptions of normality and homoscedasticity. (3) Obtain estimator properties from the flexible and robust SFAM structural model. (4) Obtaining hypothesis testing of each relationship constructed from a flexible and robust SFAM structural model. This research is integrated with a flexible and robust model approach based on nonparametric smoothing spline (RNSS) regression analysis which can capture the form of relationships that depend on empirical data, and the robustness of the model based on the distribution assumption and the assumption of homoscedasticity error variance. There are at least three transformation methods, namely SRS, MSI, and RASCH, which will be used in the development of the Structural Flexibility and Acceptance Model (SFAM). The results obtained from the research progress report are obtaining the development of a flexible structural model of the form of the relationship between variables, obtaining the development of a robust structural model of the assumptions of normality and homoscedasticity, obtaining the estimator properties of the flexible and robust SFAM structural model, and obtaining hypothesis testing. of each relationship constructed from a flexible and robust SFAM structural model. The originality of the theory in this study is very visible in the discovery of a new model, namely SFAM which can accommodate several things, which are the weaknesses of several existing analysis tools such as reciprocal and recursive models, more than one endogenous variable, flexible and robust models, overcoming inadmissible solutions, reflective indicators, formative, and reflective/formative (on the second-order), metric and non-metric data, and simultaneous processing of the input score data (through transformation to scale).
\end{abstract}

Key-Words: - Flexible and Sturdy Structural Modeling; SFAM; SEM; Disruption Innovation

Received: June 15, 2021. Revised: November 16, 2021. Accepted: November 28, 2021. Published: December 14, 2021.

\section{Introduction}

This research will reconstruct the Structural Flexibility and Acceptance Model (SFAM), as a step in developing concepts/theories/propositions regarding statistical modeling. The meaning of the 4 words in the model is as follows: structural reflects the structural modeling (system) that is built, flexibility means the flexible model, acceptance contains the meaning of a robust and assumptionfree model, and the model reflects the reconstruction of stochastic-based (probabilistic) statistical modeling. The existing statistical modeling which is widely used, such as SEM, PLS, GSCA and WarpPLS, has its weaknesses and will be improved by carrying out SFAM reconstruction. In addition, it will also be updated by adding several new statistical concepts that are often needed by statistics users. Modeling of this system is a data analysis method that is very widely used, especially in the social, humanities and education fields, in research with a quantitative approach.

Quantitative, qualitative research approaches and mixed methods in various fields develop complementarily, that is, complementary. For example, research in accounting which initially prioritized a quantitative approach, due to its scientific nature being closer to the numerical computation process, has now begun to shift to a qualitative approach, especially after developing behavioral accounting. Likewise in the field of economics. On the other hand, the scientific field, which was originally normative and empirical research with a qualitative approach, has also begun to penetrate a quantitative approach, for example by starting to develop jurimetry in research in the field of legal science. Like other social and humanities fields and have developed earlier, for example, sociometry, psychometry and econometry. 
Research with a quantitative approach in the social, humanities and education fields did not initially become an attractive alternative, considering that researchers often experience difficulties in analyzing data. The application of statistics as a method of data analysis, in general, makes it difficult for them. However, with the development of computing tools in the form of computer machines, this problem has been overcome. Research in the field of social humanities and education, in general, is investigating systems, where one of the characteristics of the system is complex. The simplification solution can be done through modeling, one of which is statistical modeling. The main characteristic of statistical modeling is that there are relationships between variables and the nature of these relationships is stochastic. Meanwhile, in some textbooks and scientists' views, the process of formulating the relationship between variables in a model still puts forward the need for a theoretical basis or the results of previous research. In other words, the design of the model always begins with the formulation of a research hypothesis.

Theory and the results of previous research can certainly be the logic of thinking that has been in the past. In designing the relationship between variables in a model that is based on the construction of a hypothesis, in principle, we bring the logic of thinking to the past (the past) for today's footing. Thus, if we are still putting this forward, then we will have difficulty making preparations for the future. This research is also based on research conducted by Iwu et al. [14], Amirudin et al. [15], and Kraus et al. [16].

The effect of disruption, bringing the logic of future thinking (besuk) to today's foothold, is one answer to this problem. We can follow the innovation of disruption if the basis or perspective of research thinking, namely in the arrangement of relationships between variables in the model, we expand it as follows: (1) The norm of finality (scripture), axioms, theorems/theories/propositions, (4) The results of empirical research, (5) The adoption of theory and/or the results of empirical research on the relationship between variables from other fields of science, (6) Non-final norms, for example, government regulations, laws, SOPs, and so on, ( 7) Empirical conditions, (8) Expert judgment, and (9) Intuition/logic. The elements (1), (6) s, d (9) allow us to construct or reconstruct the model (theory). Research novelty (new ideas/ideas) can be more easily extracted from local wisdom if the foundation or perspective of research thinking can be broadened like that. This can also encourage the development of indigenous science.

The nature of research subjects in the social, humanities, and education fields is rapidly changing and dynamic. System modeling that is based on disruption innovation is seen as being able to keep up with these fast and dynamic changes and even to catch symptoms in the future. Thus, it is hoped that researchers, students and lecturers are not confined to existing theories, but can carry out scientific constructions even though they are through quantitative research.

This research is an effort to socialize these ideas. With disruption innovation, the main emphasis is on impact, namely what we produce and have an impact on life, both scientific life and the life of society in general. Analysis of system modeling that has developed and is widely used includes Structural Equation Modeling (SEM). Until now, there have been several SEM analyzes whose applications have developed in Indonesia, including SEM with AMOS / LISREL software, PLS with SmartPLS software, GSCA with GeSCA and WarpPLS software.

The modeling involves latent variables, in which the data analyzed is generally obtained from the measurement process using a questionnaire. The questionnaire by applying an attitude scale model, for example, a Likert scale, produces data in the form of scores. In general, the weaknesses of all these methods have not included the concept of transforming data in the form of scores into scales. So it is necessary to develop a model that includes the concept of transforming data in the form of scores into scales. There are at least three transformation methods, namely SRS, MSI, and RASCH, which will be used to complement the modeling method, by developing a Structural Flexibility and Acceptance Model (SFAM).

The Structural Flexibility and Acceptance Model (SFAM) reconstruction will also be equipped with a path analysis concept with categorical data so that it can also be used to analyze categorical data. On the other hand, the development of SFAM is also equipped with a flexible and robust model approach based on nonparametric smoothing spline (RNSS) regression analysis which can capture the form of relationships that depend on empirical data, and the robustness of the model based on distribution assumptions and the assumption of homoscedasticity error variance, and data usage. longitudinal / panel, and time series. The previous description illustrates that basically, it is necessary to develop statistical modeling concepts/theories/propositions. This research is 
intended to describe a flexible and robust structural modeling, the realization of a new model, namely the Structural Flexibility and Acceptance Model (SFAM). This study aims to: (5) Develop a flexible structural model of the relationship between variables. (6) Develop a structural model that is robust with the assumptions of normality and homoscedasticity. (7) Obtain estimator properties from the flexible and robust SFAM structural model. (8) Obtaining hypothesis testing of each relationship constructed from a flexible and robust SFAM structural model.

This research was conducted to reconstruct the Structural Flexibility and Acceptance Model (SFAM), which is a robust structural model for the assumption of linearity, or in other words, a structural model that can accommodate the form of relationships between variables that are non-linear and also robust to distribution assumptions and homoscedasticity assumptions, variance error. Besides, SFAM is also equipped with the power to be used to analyze categorical data. The originality of the theory in this study is very visible in the discovery of a new model, namely SFAM which can accommodate several things, which are the weaknesses of several existing analysis tools such as reciprocal and recursive models, more than one endogenous variable, flexible and robust models, overcoming inadmissible solutions, reflective indicators, formative, and reflective/formative (on the second-order), metric and non-metric data, and simultaneous processing of the input score data (through transformation to scale).

\section{Result and Discussion}

\subsection{Path Analysis}

Path analysis is a development of regression analysis, involving more than one function. Path analysis is not a method for finding causes, but an applied method for causal models formulated by researchers based on knowledge and theoretical considerations.

In path analysis, there are at least two endogenous variables, one as an intervening endogenous variable and one as a pure endogenous variable. The emergence of complex problems because as many as $\mathrm{k} \geq 2$ endogenous variables will appear $\mathrm{k}$ equations in which the equations will be interrelated or correlated. Path analysis is based on the assumption of linearity, namely a linear relationship between 2 or more variables.

The linear model can only be applied to linear problems which are characterized by a straight curve of the relationship between exogenous variables and endogenous variables. When the linearity assumption is not met, an alternative method is developed to overcome this problem, namely the nonlinear path model. Similar to nonlinear regression, nonlinear path analysis is a method to obtain a nonlinear model that expresses the relationship of exogenous variables and endogenous variables, whose pattern does not follow a straight line, but follows a certain curve shape, such as quadratic, cubic, polynomial degree k, logarithm, exponential, and so on.

In nonlinear regression the regression model is assumed that the pattern of the relationship between the response variable and the predictor variable can be described in a certain function. Linear or nonlinear relationship between predictor variables and response variables can be known through testing, one of which is using the Ramsey's RESET test. The RESET approach utilizes OLS to minimize the number of errors squared from each observation (Gujarati, 2004). The following is the linearity test procedure using RESET:

There are other weaknesses in path analysis, including the measurement model is carried out outside the path analysis model, the relationship between variables cannot be reciprocal, and the form of the model that can be accommodated in the model is only a linear model (not flexible).

A model is said to be valid if the underlying assumptions have been met. In addition, according to two indicators of model validity in the path analysis, namely (1) the Total Determination Coefficient (KDT) which ranges from $0 \%$ to $100 \%$ and (2) Trimming Theory, which uses the probability value of the test, namely the regression coefficient test. partially standardized. Theory Trimming is an approach to improve the path analysis model by removing path coefficients that do not meet the significance criteria. 


\subsection{Development of a Flexible Structural Model of the Form of Relationship between Variables}

Regression analysis is a function of the exogenous variable $(\mathrm{X})$ on the endogenous variable $(\mathrm{Y})$ which can be either linear or non-linear. The simple linear regression analysis has one exogenous variable and one endogenous variable with the form of a relationship that can be described as a straight line (linear). Meanwhile, simple quadratic regression analysis is a function of one endogenous variable and one exogenous variable which has the highest rank equal to two, so that the shape of the relationship can be described as a parabolic line.

Path analysis is an extension of multiple regression analysis, wherein the simple path analysis, there is one exogenous variable and two endogenous variables involved, namely the intervening endogenous variable (Y1) and the pure endogenous variable (Y2) which can be described following the simple path analysis model in Figure 1.

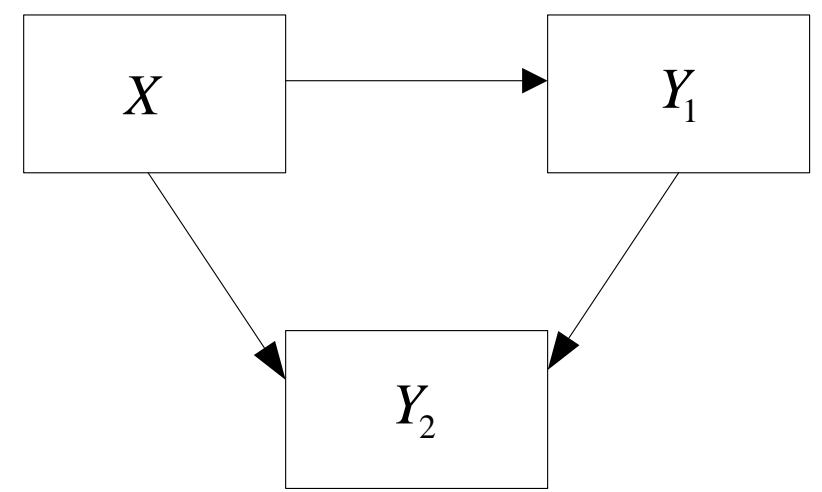

Fig. 1: Simple Path Analysis Diagram

Just like regression analysis, path analysis can also describe the pattern of relationships between variables that are linear and non-linear. Simple quadratic path analysis involves one exogenous variable and one intervening endogenous variable of order $\mathrm{m}=2$, and one pure endogenous variable of order $\mathrm{m}=1$. Meanwhile, complex linear path analysis involves one exogenous variable with $\mathrm{q}$ the endogenous variable. All variables in complex linear path analysis have an order of $\mathrm{m}=1$.Thus, a complex quadratic path analysis model can be made with all variables of order $m=2$. The path analysis model can be expanded again by adding an order of $\mathrm{m}$ for each exogenous and endogenous intervening variable so that can produce a simple m-order path analysis model (with 2 endogenous variables) and complex m-order path analysis (with q-1 endogenous variables and q-1 intervening endogenous variables).

\section{Lemma 2.1 Form of Simple Quadratic Path} Analysis Model

If given paired data $\left(X_{i}, Y_{1 i}, Y_{2 i}\right)$ with $i=1,2,3, \ldots, n$ following the quadratic path analysis model, the quadratic path analysis function is obtained as presented in equation (1) and the model (2).

$Y_{1 i}=f\left(X_{i}, X_{i}^{2}\right)+\varepsilon_{1 i}$

$Y_{2 i}=f\left(X_{i}, X_{i}^{2}, Y_{1 i}, Y_{1 i}^{2}\right)+\varepsilon_{2 i}$

$Y_{1 i}=\beta_{011}+\beta_{111} X_{i}+\beta_{211} X_{i}^{2}+\varepsilon_{1 i}$

$Y_{2 i}=\beta_{012}+\beta_{112} X_{i}+\beta_{212} X_{i}^{2}+\beta_{122} Y_{1 i}+\beta_{222} Y_{1 i}^{2}+\varepsilon_{2 i}$

with the form of a matrix

${\underset{\sim}{Y}}_{2 n \times 1}=\mathbf{X}_{2 n \times 8} \beta_{\sim \times 1}+\varepsilon_{\sim 2 n \times 1}$

\section{Proof :}

Before obtaining the model in the Simple Quadratic Path Analysis, the first model is obtained from (a) Simple Linear Regression Analysis; (b) Simple Linear Path Analysis; and (c) Quadratic Regression Analysis as follows:

First part: Find out the simple linear regression model with equation (3) and the model in (4).

$$
\begin{gathered}
Y_{1 i}=f\left(X_{i}\right)+\varepsilon_{1 i} \\
Y_{i}=\beta_{0}+\beta_{1} X_{i}+\varepsilon_{i}
\end{gathered}
$$

The matrix can be formed:

$Y_{\sim \times 1}=\mathbf{X}_{n \times 2} \beta_{\sim 2 \times 1}+{\underset{\sim}{n \times 1}}_{n \times 1}$

$\left[\begin{array}{c}Y_{1} \\ Y_{2} \\ Y_{3} \\ \vdots \\ Y_{n}\end{array}\right]=\left[\begin{array}{cc}1 & X_{1} \\ 1 & X_{2} \\ 1 & X_{3} \\ \vdots & \vdots \\ 1 & X_{n}\end{array}\right]\left[\begin{array}{l}\beta_{0} \\ \beta_{1}\end{array}\right]+\left[\begin{array}{c}\varepsilon_{1} \\ \varepsilon_{2} \\ \varepsilon_{3} \\ \vdots \\ \varepsilon_{n}\end{array}\right]$,

Where:

$Y_{i}$ : Endogenous variable of the $i$-th observation $(i=1,2,3, \ldots, n)$;

$X_{i}$ : Exogenous variable of the $i$-th observation;

$n$ : The number of observations;

$\beta_{j}$ : The coefficient of influence of exogenous variables on endogenous $(j=0,1,2)$;

$\varepsilon_{i}$ : Random error of the $i$ endogenous variable

Part Two: Find a simple linear path analysis model as presented in equation (5) and the model in (6).

$Y_{1 i}=f\left(X_{i}\right)+\varepsilon_{1 i}$

$Y_{2 i}=f\left(X_{i}, Y_{1 i}\right)+\varepsilon_{2 i}$ 
$Y_{1 i}=\beta_{01}+\beta_{11} X_{i}+\varepsilon_{1 i}$

$Y_{2 i}=\beta_{02}+\beta_{12} X_{i}+\beta_{22} Y_{1 i}+\varepsilon_{2 i}$

The following matrix can be formed:

$Y_{\sim 2 n \times 1}=\mathbf{X}_{2 n \times 5} \beta_{5 \times 1}+\varepsilon_{\sim 2 n \times 1}$

Part Three: After knowing the equations and simple linear regression models, a simple quadratic regression model can be made as presented in equations (7) and (8).

$Y_{i}=f\left(X_{i}, X_{i}^{2}\right)+\varepsilon_{i}$

$Y_{i}=\beta_{0}+\beta_{1} X_{i}+\beta_{2} X_{i}^{2}+\varepsilon_{i}$

The matrix form of the model is:

$Y_{\sim n 1}=\mathbf{X}_{n \times 3} \beta_{\sim \times 1}+\varepsilon_{\sim n \times 1}$

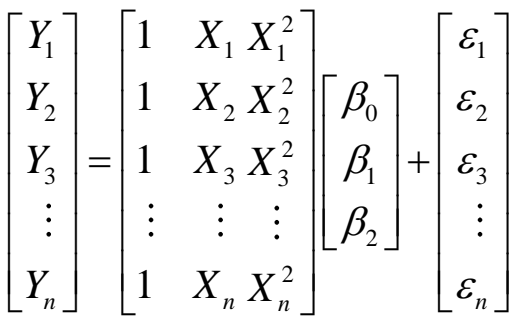

Where:

$Y_{i} \quad$ : Endogenous variable of the $i$-th observation

$$
(i=1,2,3, \ldots, n) \text {; }
$$

$X_{i}$ : Exogenous variable of the $i$-th observation;

$X_{i}^{2}$ : Exogenous variable to the power of 2

(squared) of the $i$-th observation;

$n$ : The number of observations;

$q$ : The number of exogenous variables;

$\beta_{j}$ : The coefficient of influence of exogenous variables on endogenous $(j=0,1,2)$;

$\varepsilon_{i} \quad$ : Random error of the $i$ endogenous variable

$Y_{1 i}=\beta_{011}+\beta_{111} X_{i}+\beta_{211} X_{i}^{2}+\varepsilon_{1 i}$

$Y_{2 i}=\beta_{012}+\beta_{112} X_{i}+\beta_{212} X_{i}^{2}+\beta_{122} Y_{1 i}+\beta_{222} Y_{1 i}^{2}+\varepsilon_{2 i}$

$Y_{3 i}=\beta_{013}+\beta_{113} X_{i}+\beta_{213} X_{i}^{2}+\beta_{123} Y_{1 i}+\beta_{223} Y_{1 i}^{2}+\beta_{133} Y_{2 i}+\beta_{233} Y_{2 i}^{2}+\varepsilon_{3 i}$

$Y_{(q-1) i}=\beta_{01(q-1)}+\beta_{11(q-1)} X_{i}+\beta_{21(q-1)} X_{i}^{2}+\beta_{12(q-1)} Y_{1 i}+\beta_{22(q-1)} Y_{1 i}^{2}+\beta_{13(q-1)} Y_{2 i}+\beta_{23(q-1)} Y_{2 i}^{2}+\cdots+\beta_{1(q-1)(q-1)} Y_{q-2, i}+\beta_{2(q-1)(q-1)} Y_{q-2, i}^{2}+\varepsilon_{(q-1) t}$

$Y_{q i}=\beta_{01 q}+\beta_{11 q} X_{i}+\beta_{21 q} X_{i}^{2}+\beta_{12 q} Y_{1 i}+\beta_{22 q} Y_{1 i}^{2}+\beta_{13 q} Y_{2 i}+\beta_{23 q} Y_{2 i}^{2}+\cdots+\beta_{1 q q} Y_{q-1, i}+\beta_{2 q q} Y_{q-1, i}^{2}+\varepsilon_{q i}$

With the form of a matrix:

$Y_{\sim q n \times 1}=\mathbf{X}_{q n \times\left(q+\Sigma_{i=1}^{q} 1^{i}\right)} \underset{\sim\left(q+\sum_{i=1}^{q} i\right) \times 1}{ }+{\underset{\sim}{q n \times 1}}_{q}$

Proof :

First part: The equations and models in the simple quadratic path analysis are known as shown in equations (11) and (12).

$Y_{1 i}=f\left(X_{i}, X_{i}^{2}\right)+\varepsilon_{1 i}$

$Y_{2 i}=f\left(X_{i}, X_{i}^{2}, Y_{1 i}, Y_{1 i}^{2}\right)+\varepsilon_{2 i}$
From the equations in the simple linear regression analysis model, simple path analysis, and quadratic regression analysis that has been described, a function that is formed as in equations (1) and (2) can be obtained, so that the following matrix is obtained:

$Y_{2 n \times 1}=\mathbf{X}_{2 n \times 8} \beta_{\sim \times 1}+\varepsilon_{\sim 2 n \times 1}$

$\left[\begin{array}{c}Y_{11} \\ Y_{12} \\ Y_{13} \\ \vdots \\ Y_{1 n} \\ Y_{21} \\ Y_{22} \\ Y_{23} \\ \vdots \\ Y_{2 n}\end{array}\right]=\left[\begin{array}{cc}\mathbf{X}_{X X^{2}} & \mathbf{0}_{n \times 5} \\ \mathbf{0}_{n \times 3} & \mathbf{X}_{X X^{2} Y Y^{2}}\end{array}\right]\left[\begin{array}{c}\beta_{011} \\ \beta_{111} \\ \beta_{211} \\ \beta_{012} \\ \beta_{112} \\ \beta_{212} \\ \beta_{122} \\ \beta_{222}\end{array}\right]+\left[\begin{array}{c}\varepsilon_{11} \\ \varepsilon_{12} \\ \varepsilon_{13} \\ \vdots \\ \varepsilon_{1 n} \\ \varepsilon_{21} \\ \varepsilon_{22} \\ \varepsilon_{23} \\ \vdots \\ \varepsilon_{2 n}\end{array}\right]$

\section{Lemma 2.2 Form of Complex Quadratic Path} Analysis Model

If given paired data $\left(X_{i}, Y_{1 i}, Y_{2 i}, Y_{3 i}, \ldots, Y_{(q-1) i}, Y_{q i}\right)$, equations and complex quadratic path analysis models can be made as presented in (9) and (10).

$Y_{1 i}=f\left(X_{i}, X_{i}^{2}\right)+\varepsilon_{1 i}$

$Y_{2 i}=f\left(X_{i}, X_{i}^{2}, Y_{1 i}, Y_{1 i}^{2}\right)+\varepsilon_{2 i}$

$Y_{3 i}=f\left(X_{i}, X_{i}^{2}, Y_{1 i}, Y_{1 i}^{2}, Y_{2 i}, Y_{2 i}^{2}\right)+\varepsilon_{3 i}$

$Y_{(q-1) i}=f\left(X_{i}, X_{i}^{2}, Y_{1 i}, Y_{1 i}^{2}, Y_{2 i}, Y_{2 i}^{2}, \ldots, Y_{q-2, i}, Y_{q-2, i}^{2}\right)+\varepsilon_{(q-1) i}$

$Y_{q i}=f\left(X_{i}, X_{i}^{2}, Y_{1 i}, Y_{1 i}^{2}, Y_{2 i}, Y_{2 i}^{2}, \ldots, Y_{q-1, i}, Y_{q-1, i}^{2}\right)+\varepsilon_{q i}$

$Y_{1 i}=\beta_{011}+\beta_{111} X_{i}+\beta_{211} X_{i}^{2}+\varepsilon_{1 i}$

$Y_{2 i}=\beta_{012}+\beta_{112} X_{i}+\beta_{212} X_{i}^{2}+\beta_{122} Y_{1 i}+\beta_{222} Y_{1 i}^{2}+\varepsilon_{2 i}$

The matrix form of the model is:

$Y_{\sim 2 n \times 1}=\mathbf{X}_{2 n \times 8} \beta_{8 \times 1}+\varepsilon_{\sim 2 n \times 1}$

Part Two: Known equations and models in complex linear path analysis as shown in equations (13) and (14). 
$Y_{1 i}=f\left(X_{i}\right)+\varepsilon_{1 i}$

$Y_{2 i}=f\left(X_{i}, Y_{1 i}\right)+\varepsilon_{2 i}$

$Y_{3 i}=f\left(X_{i}, Y_{1 i}, Y_{2 i}\right)+\varepsilon_{3 i}$

$\vdots$

$Y_{q i}=f\left(X_{i}, Y_{1 i}, Y_{2 i}, \ldots, Y_{q-1, i}\right)+\varepsilon_{q i}$

$Y_{1 i}=\beta_{01}+\beta_{11} X_{i}+\varepsilon_{1 i}$

$Y_{2 i}=\beta_{02}+\beta_{12} X_{i}+\beta_{22} Y_{11}+\varepsilon_{2 i}$

$Y_{3 i}=\beta_{03}+\beta_{13} X_{i}+\beta_{23} Y_{21}+\beta_{33} Y_{22}+\varepsilon_{3 i}$

$Y_{q i}=\beta_{0 q}+\beta_{1 q} X_{i}+\beta_{2 q} Y_{q 1}+\beta_{3 q} Y_{q 2}+\cdots+\beta_{q q} Y_{q, q-1}+\varepsilon_{q i}$

The matrix form of the model is:

$Y_{\sim q n \times 1}=\mathbf{X}_{q n \times\left(q+\Sigma_{i=1}^{q} i\right)} \underset{\sim\left(q+\sum_{i=1}^{q} i\right) \times 1}{\beta}+{\underset{\sim}{q} q n \times 1}$

From the results of the description of the model above, an extension of the model obtained in
Lemma 3.1 can be made into a complex quadratic path analysis model following equation (9) and the model in (10), to obtain the following matrix form:

$Y_{\sim q n \times 1}=\mathbf{X}_{\left.q n \times\left(q+\sum_{i=1}^{q}\right)^{i}\right)} \beta_{\sim\left(q+\Sigma_{i=1}^{q} i\right) \times 1}+{\underset{\sim}{\sim q n \times 1}}$

Lemma 2.3 Form of Simple Non-Linear Path Analysis Model (non-linear m-order)

If paired data $\left(X_{i}, Y_{1 i}, Y_{2 i}\right)$ with $i=1,2,3, \ldots, n$ follows a simple $m$-order path analysis model, equations and models can be obtained as presented in equations (15) and (16).

$$
\begin{aligned}
& Y_{1 i}=f\left(X_{i}, X_{i}^{2}, X_{i}^{3}, \ldots, X_{i}^{m}\right)+\varepsilon_{1 i} \\
& Y_{2 i}=f\left(X, X_{i}^{2}, X_{i}^{3}, \ldots, X^{m}, Y_{1 i}, Y_{1 i}^{2}, Y_{1 i}^{3}, \ldots, Y_{1 i}^{m}\right)+\varepsilon_{2 i}
\end{aligned}
$$

The matrix form of the model is:

$Y_{\sim 2 n \times 1}=\mathbf{X}_{2 n \times(3 m+2)} \beta_{\sim(3 m+2) \times 1}+{\underset{\sim}{2 n \times 1}}$

Proof :

Part One: The results of Lemma 2.1 have been obtained which are a form of the quadratic path analysis function as presented in equation (2.1.1) and the model in (2.1.2) with the following matrix form:

${\underset{\sim}{2 n \times 1}}_{\sim}=\mathbf{X}_{2 n \times 8} \beta_{\sim} \beta_{8 \times 1}+{\underset{\sim}{2 n \times 1}}_{2}$

$\left[\begin{array}{c}Y_{11} \\ Y_{12} \\ Y_{13} \\ \vdots \\ Y_{1 n} \\ Y_{21} \\ Y_{22} \\ Y_{23} \\ \vdots \\ Y_{2 n}\end{array}\right]=\left[\begin{array}{cc}\mathbf{X}_{X X^{2}} & \mathbf{0}_{n \times 5} \\ \mathbf{0}_{n \times 3} & \mathbf{X}_{X X^{2} Y Y^{2}}\end{array}\right]\left[\begin{array}{c}\beta_{011} \\ \beta_{111} \\ \beta_{211} \\ \beta_{012} \\ \beta_{112} \\ \beta_{212} \\ \beta_{122} \\ \beta_{222}\end{array}\right]+\left[\begin{array}{c}\varepsilon_{11} \\ \varepsilon_{12} \\ \varepsilon_{13} \\ \vdots \\ \varepsilon_{1 n} \\ \varepsilon_{21} \\ \varepsilon_{22} \\ \varepsilon_{23} \\ \vdots \\ \varepsilon_{2 n}\end{array}\right]$

From the results in Lemma 2.1, an expansion can be made by adding the order to $m$ in the exogenous and endogenous intervening variables, so that equations and models are obtained as presented in equations (15) and (16) above with the following matrix form: ${\underset{\sim}{2 n \times 1}}_{1}=\mathbf{X}_{2 n \times(3 m+2)} \beta_{\sim(3 m+2) \times 1}+{\underset{\sim}{2 n \times 1}}_{2}$
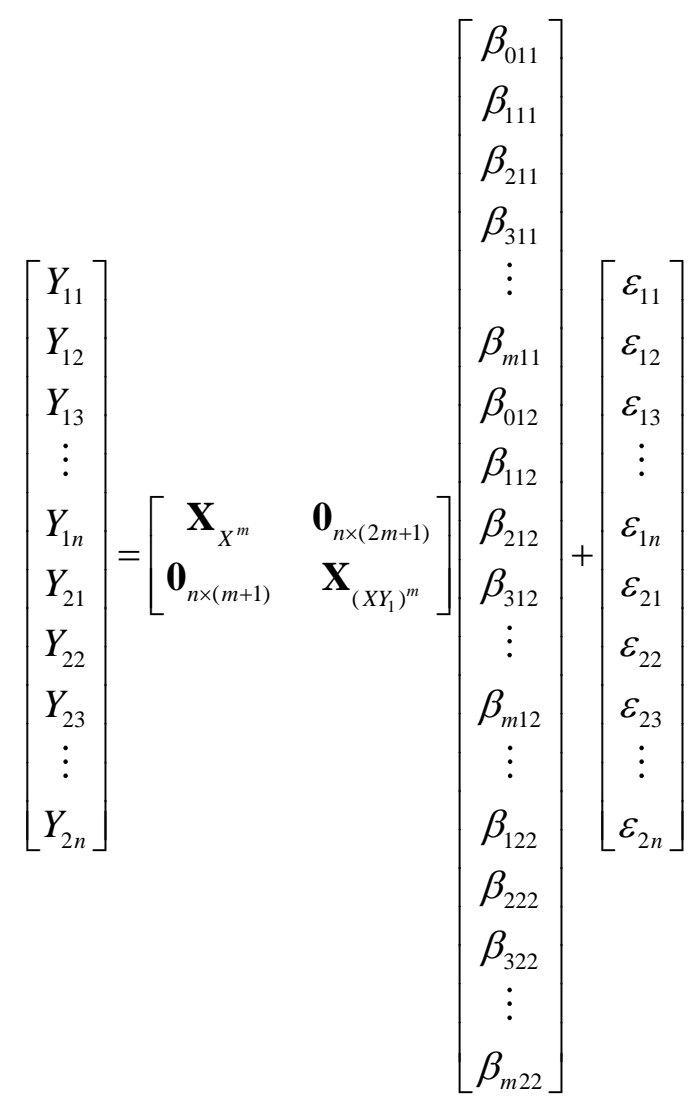

Lemma 2.4 Model Shape Non-Linear Complex Path Analysis (non-linear m-order) 
If given paired data $\left(X_{i}, Y_{1 i}, Y_{2 i}, Y_{3 i}, \ldots, Y_{q i}\right)$ with $i=1,2,3, \ldots, n$ which follows a complex non-linear path analysis model of order $\mathrm{m}$, we can obtain the $Y_{1 i}=f\left(X, X^{2}, X^{3}, \ldots, X^{m}\right)+\varepsilon_{1 i}$

$Y_{2 i}=f\left(X, X^{2}, X^{3}, \ldots, X^{m}, Y_{1 i}, Y_{1 i}^{2}, Y_{1 i}^{3}, \ldots, Y_{1 i}^{m}\right)+\varepsilon_{2 i}$

$\vdots$

$Y_{q i}=f\left(X, X^{2}, X^{3}, \ldots, X^{m}, Y_{1 i}, Y_{1 i}^{2}, Y_{1 i}^{3}, \ldots, Y_{1 i}^{m}, \ldots, \mathrm{Y}_{q-1, i}, \mathrm{Y}_{q-1, i}^{2}, \mathrm{Y}_{q-1, i}^{3}, \ldots, \mathrm{Y}_{q-1, i}^{m}\right)+\varepsilon_{q i}$

$Y_{1 i}=\beta_{011}+\beta_{111} X_{i}+\beta_{211} X_{i}^{2}+\cdots+\beta_{m 11} X_{i}^{m}+\varepsilon_{1 i}$

$Y_{2 i}=\beta_{012}+\beta_{112} X_{i}+\beta_{212} X_{i}^{2}+\cdots+\beta_{m 12} X_{i}^{m}+\beta_{122} Y_{1 i}+\beta_{222} Y_{1 i}^{2}+\cdots+\beta_{m 22} Y_{1 i}^{m}+\varepsilon_{2 i}$

$Y_{q i}=\beta_{01 q}+\beta_{11 q} X_{i}+\beta_{21 q} X_{i}^{2}+\cdots+\beta_{m 1 q} X_{i}^{m}+\beta_{12 q} Y_{1 i}+\beta_{22 q} Y_{1 i}^{2}+\cdots+\beta_{m 2 q} Y_{1 i}^{m}+\cdots+\beta_{1 q q} Y_{q-1, i}+\beta_{2 q q} Y_{q-1, i}^{2}+\cdots+\beta_{m q q} Y_{q-1, i}^{m}+\varepsilon_{q i}$ and (18)

The matrix form of the model is:

$Y_{\sim q n \times 1}=\mathbf{X}_{\left.q n \times\left(q+m \Sigma_{i=1}^{q}\right)^{i}\right)} \beta_{\sim\left(q+m \Sigma_{i=1}^{q} i\right) \times 1}+{\underset{\sim}{q n \times 1}}$

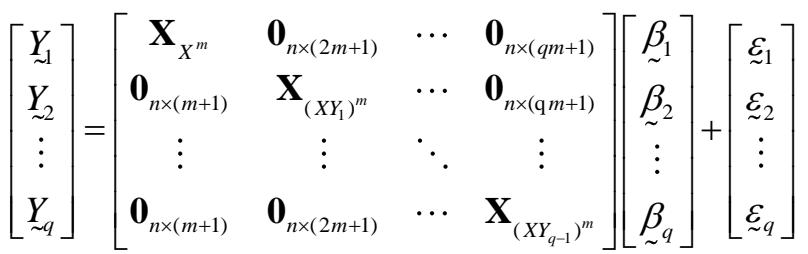

\section{Proof :}

The first part: The equations and models in the complex quadratic path analysis are known as presented in (9) and (10) with the following matrix form :

$Y_{\sim q n \times 1}=\mathbf{X}_{q n \times\left(q+\sum_{i=1}^{q} i\right)} \underset{\sim\left(q+\Sigma_{i=1}^{q} i\right) \times 1}{\beta}+\mathcal{\varepsilon}_{q n \times 1}$

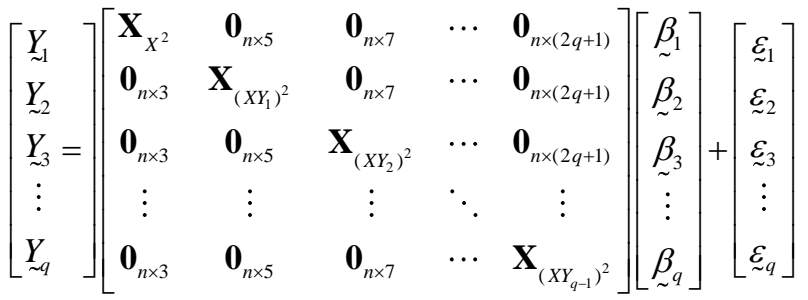

The second part: Know the equations and models in the simple non-linear path analysis of order $m$ as presented in equation (14) and model (15) with the following matrix form:

${\underset{\sim}{2 n \times 1}}=\mathbf{X}_{2 n \times(3 m+2)}{\underset{\sim}{(3 m+2) \times 1}}+{\underset{\sim}{2 n \times 1}}_{2 n}$

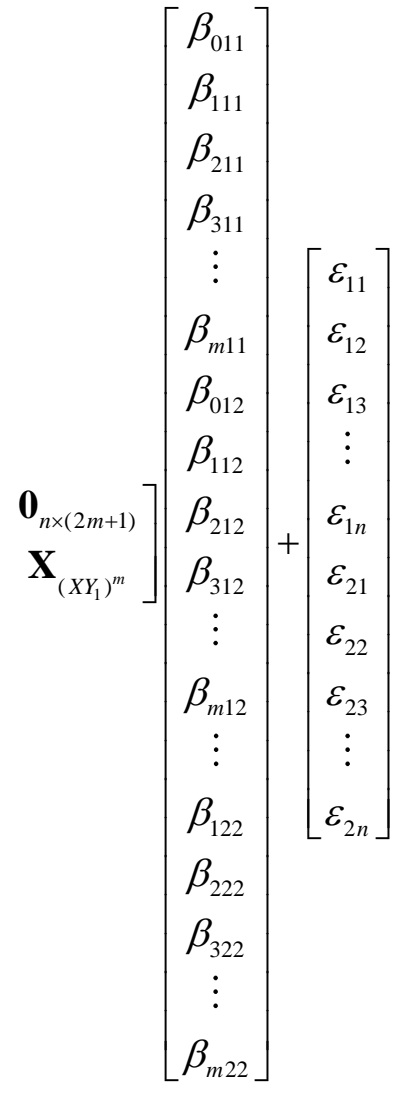




\subsection{Development of Structural Models that are Strong with Assumptions of Normality and Homoscedasticity}

In a linear model in parameters, the Ordinary Least Square (OLS) method can be used by minimizing the number of squares of the residuals to estimate the path coefficients of the general form of the matrix operation. $\underset{\%}{Y}=\mathbf{X} \underset{\sigma \text { \% }}{\beta}+\underset{\%}{\varepsilon}$, Where $\underset{\%}{\varepsilon}=\underset{\%}{Y}-\mathbf{X} \underset{\text { \% }}{\beta}$ The OLS method minimizes the following functions:

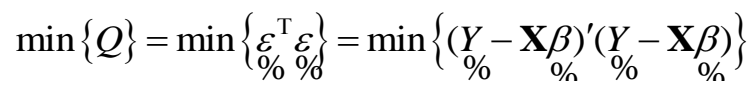

Estimation of parameters using the OLS approach by minimizing $Q$ following:

$$
\begin{aligned}
& \left.Q=\left(\begin{array}{l}
\boldsymbol{\varepsilon}^{\mathrm{T}} \boldsymbol{\varepsilon} \\
\% \%
\end{array}\right)=(\underset{\%}{Y}-\mathbf{X} \beta)^{\prime}\right)^{\prime}(\underset{\%}{Y}-\mathbf{X} \beta \underset{\%}{\beta}) \\
& =\left(\underset{\%}{Y_{\%}^{\prime}}-\mathbf{X}^{\prime} \beta_{\%}^{\prime}\right)(\underset{\%}{Y}-\mathbf{X} \underset{\%}{\beta})
\end{aligned}
$$

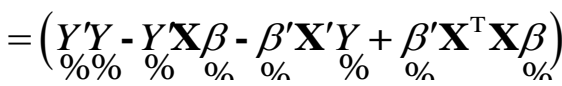

$$
\begin{aligned}
& =\left(\underset{\% \%}{Y_{\%}^{\prime} Y}-2 \underset{\%}{\beta^{\prime} \mathbf{X}^{\prime}} \underset{\%}{Y}+\beta_{\%}^{\prime} \mathbf{X}^{\prime} \mathbf{X} \beta\right)
\end{aligned}
$$

The solution to the optimization of equation (19) by doing the derivative $Q$ to $\beta$ and equated with 0 .

$$
\begin{aligned}
& \frac{\partial(Q)}{\partial(\theta)}=0 \\
& \% \\
& -2 \mathbf{X}^{\prime} Y+2 \mathbf{X}^{\prime} \mathbf{X} \hat{\beta}=0 \\
& \mathbf{X}_{\%}^{\prime} Y_{\%}+\mathbf{X}^{\prime} \mathbf{X} \hat{\beta}=0 \\
& \mathbf{X}_{\%}^{\prime} \mathbf{X} \hat{\beta}=\mathbf{X}_{\%}^{\prime}{ }_{\%} \\
& \hat{\beta}=\left(\mathbf{X}^{\prime} \mathbf{X}\right)^{-1} \mathbf{X}_{\%}^{\prime} Y_{\%}
\end{aligned}
$$

\section{Theorem 2.2.2 WLS}

In the following section, the prediction process will be carried out $\hat{\beta}$ using a Weighted Least Square (WLS) optimization which can accommodate the correlation between errors using a weighted (weighted) in the form of an inverse of the error covariance variance matrix, namely by solving the equation with the value $\mathbf{X}$ and $\beta$. The equation is as follows:

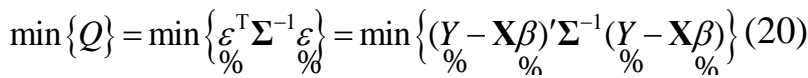
To solve the optimization in equation (20), a partial derivative is performed, as follows:

$$
\begin{aligned}
& Q(\underset{\%}{\beta})=(\underset{\%}{Y}-\mathbf{X} \beta)^{\prime} \mathbf{\Sigma}^{-1}(\underset{\%}{Y}-\mathbf{X} \beta)=\left(\underset{\%}{\gamma_{0}^{\prime}}-\beta_{\%}^{\beta^{\prime}} \mathbf{X}^{\prime}\right) \boldsymbol{\Sigma}^{-1}(\underset{\%}{Y}-\mathbf{X} \beta)
\end{aligned}
$$

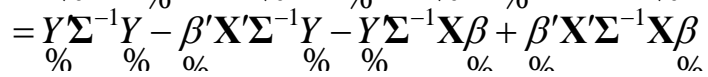

$$
\begin{aligned}
& =Y_{\%} \boldsymbol{\Sigma}^{-1} Y_{\%}-2 \beta^{\prime} \mathbf{X}^{\prime} \boldsymbol{\Sigma}^{-1} Y_{\%}+\beta_{\text {\% }}^{\prime} \mathbf{X}^{\prime} \boldsymbol{\Sigma}^{-1} \mathbf{X} \beta
\end{aligned}
$$

Note that $Y_{O I_{-}} \mathbf{X} \beta=\beta^{\prime} \mathbf{X}^{\prime} Y_{O I_{-}}$because the result of both is scalar. The next process is to derive equation (16) for $\beta$ yields equation (17). After that it is derived for $\beta$, then equalized to zero can be seen in equation (18). So that we get estimators $\beta$ in in equation (19).

$$
\begin{aligned}
& \frac{\partial Q(\beta)}{\partial \beta}=-2 \mathbf{X}^{\prime} \boldsymbol{\Sigma}^{-1}{ }_{\%}^{Y}+2 \mathbf{X}^{\prime} \boldsymbol{\Sigma}^{-1} \mathbf{X} \beta \\
& \mathbf{X}^{\prime} \boldsymbol{\Sigma}^{-1} Y_{\%}=\mathbf{X}^{\prime} \boldsymbol{\Sigma}^{-1} \mathbf{X} \hat{\beta} \\
& \hat{\beta}=\left(\mathbf{X}^{\prime} \boldsymbol{\Sigma}^{-1} \mathbf{X}\right)^{-1} \mathbf{X}^{\prime} \boldsymbol{\Sigma}^{-1}{ }_{\%}
\end{aligned}
$$

with the variance-covariance matrix $\operatorname{Var}\left(\underset{g_{-}}{\varepsilon}\right)=\Sigma$ as follows:

$$
\boldsymbol{\Sigma}=\left[\begin{array}{cccr}
\boldsymbol{\Sigma}_{11} & \boldsymbol{\Sigma}_{12} & \mathrm{~L} & \boldsymbol{\Sigma}_{1 q} \\
\boldsymbol{\Sigma}_{21} & \boldsymbol{\Sigma}_{22} & \mathrm{~L} & (2 \boldsymbol{\mathbf { L }})_{1 q} \\
\mathrm{M} & \mathrm{M} & \mathrm{O} & \mathrm{M} \\
\boldsymbol{\Sigma}_{q 1} & \boldsymbol{\Sigma}_{q 2} & \mathrm{~L} & \boldsymbol{\Sigma}_{q q}
\end{array}\right]_{(q n) \times(q n)}
$$

Where

$$
\begin{gathered}
\boldsymbol{\Sigma}_{k k}=\left[\begin{array}{cccc}
\sigma_{k 1}^{2} & 0 & \mathrm{~L} & 0 \\
0 & \sigma_{k 2}^{2} & \mathrm{~L} & 0 \\
\mathrm{M} & \mathrm{M} & \mathrm{O} & \mathrm{M} \\
0 & 0 & \mathrm{~L} & \sigma_{k n}^{2}
\end{array}\right]_{n \times n} \\
\boldsymbol{\Sigma}_{k j}=\left[\begin{array}{cccc}
\sigma_{1(\mathrm{k}, \mathrm{j})} & 0 & \mathrm{~L} & 0 \\
0 & \sigma_{2(\mathrm{k}, \mathrm{j})} & \mathrm{L} & 0 \\
\mathrm{M} & \mathrm{M} & \mathrm{O} & \mathrm{M} \\
0 & 0 & \mathrm{~L} & \sigma_{n(k, j)}
\end{array}\right]_{n \times n}
\end{gathered}
$$

and

Theorems 2.1 and 2.2 can be applied to the model respectively: 


\section{Lemma 2.1 which has a shape}

$\hat{\beta}=\left[\begin{array}{l}\hat{\beta}_{011} \\ \hat{\beta}_{111} \\ \hat{\beta}_{211} \\ \hat{\beta}_{012} \\ \hat{\beta}_{112} \\ \hat{\beta}_{212} \\ \hat{\beta}_{122} \\ \hat{\beta}_{222}\end{array}\right]$

With $\underset{\%}{Y}=\left[\begin{array}{c}Y_{11} \\ Y_{12} \\ Y_{13} \\ \mathrm{M} \\ Y_{1 n} \\ Y_{21} \\ Y_{22} \\ Y_{23} \\ \mathrm{M} \\ Y_{2 n}\end{array}\right] ; \mathbf{X}=\left[\begin{array}{cc}\mathbf{X}_{X X^{2}} & \mathbf{0}_{n \times 5} \\ \mathbf{0}_{n \times 3} & \mathbf{X}_{X X^{2} Y Y^{2}}\end{array}\right]$

Where $\mathbf{X}_{X X^{2}}=\left[\begin{array}{ccc}1 & X_{1} & X_{1}^{2} \\ 1 & X_{2} & X_{2}^{2} \\ 1 & X_{3} & X_{3}^{2} \\ \mathrm{M} & \mathrm{M} & \mathrm{M} \\ 1 & X_{n} & X_{n}^{2}\end{array}\right]$

$\mathbf{X}_{X X^{2} Y Y^{2}}=\left[\begin{array}{ccccc}1 & X_{1} & X_{1}^{2} & Y_{11} & Y_{11}^{2} \\ 1 & X_{2} & X_{2}^{2} & Y_{12} & Y_{12}^{2} \\ 1 & X_{3} & X_{3}^{2} & Y_{13} & Y_{13}^{2} \\ \mathrm{M} & \mathrm{M} & \mathrm{M} & \mathrm{M} & \mathrm{M} \\ 1 & X_{n} & X_{n}^{2} & Y_{1 n} & Y_{1 n}^{2}\end{array}\right]$ 


\section{Lemma 2.2 which has a shape}

$$
\begin{aligned}
& \hat{\beta}=\left[\begin{array}{c}
\hat{\beta}_{1} \\
\% \\
\hat{\beta}_{2} \\
\% \\
\hat{\beta}_{3} \\
\% \\
\mathrm{M} \\
\hat{\beta}_{q} \\
\text { of }_{h}
\end{array}\right] \\
& \text { with } \%=\left[\begin{array}{c}
Y \\
\% \\
Y \\
\% \\
Y \\
\% \\
\mathrm{M} \\
Y \\
\%
\end{array}\right] \\
& \mathbf{X}=\left[\begin{array}{ccccc}
\mathbf{X}_{X^{2}} & \mathbf{0}_{n \times 5} & \mathbf{0}_{n \times 7} & \mathrm{~L} & \mathbf{0}_{n \times(2 q+1)} \\
\mathbf{0}_{n \times 3} & \mathbf{X}_{\left(X Y_{1}\right)^{2}} & \mathbf{0}_{n \times 7} & \mathrm{~L} & \mathbf{0}_{n \times(2 q+1)} \\
\mathbf{0}_{n \times 3} & \mathbf{0}_{n \times 5} & \mathbf{X}_{\left(X Y_{2}\right)^{2}} & \mathrm{~L} & \mathbf{0}_{n \times(2 q+1)} \\
\mathrm{M} & \mathrm{M} & \mathrm{M} & \mathrm{O} & \mathrm{M} \\
\mathbf{0}_{n \times 3} & \mathbf{0}_{n \times 5} & \mathbf{0}_{n \times 7} & \mathrm{~L} & \mathbf{X}_{\left(X Y_{q-1}\right)^{2}}
\end{array}\right]
\end{aligned}
$$

\section{Lemma 2.3 which has a shape}

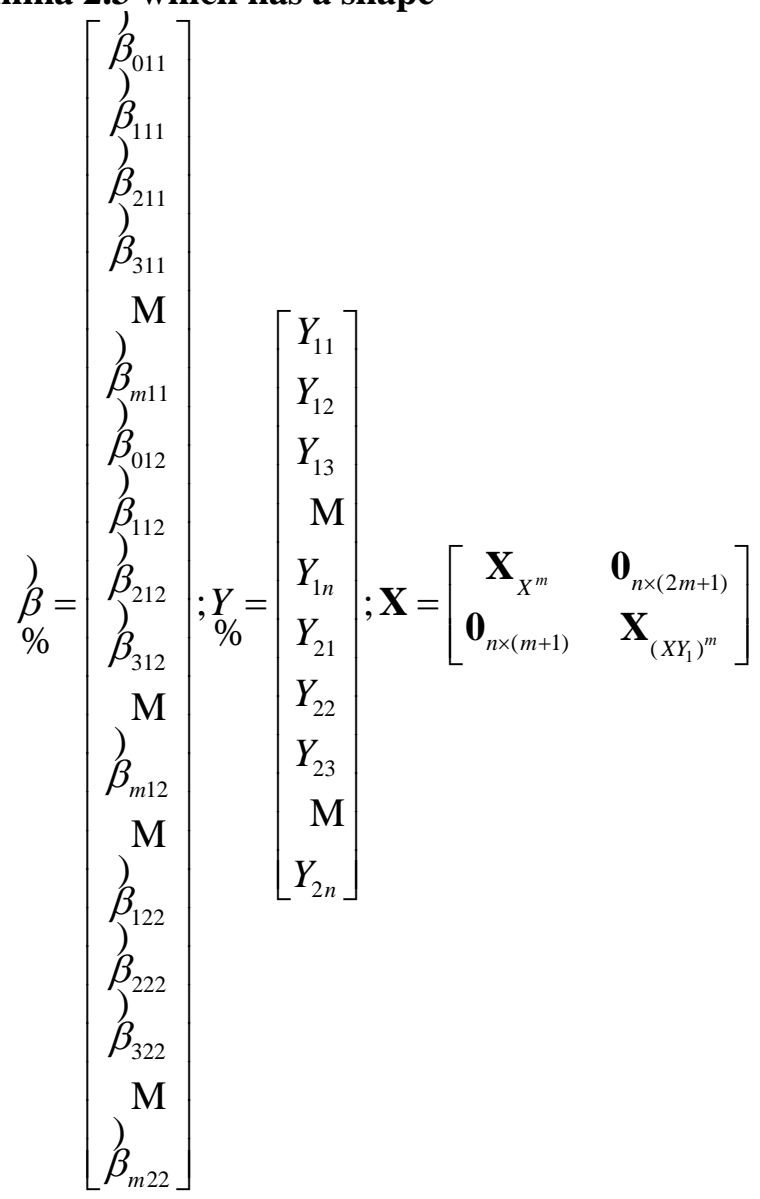

\section{Lemma 2.4 which has a shape}

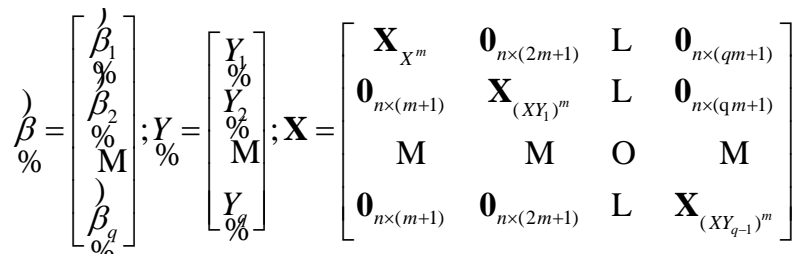

\section{Linearity Testing}

The linearity test aims to determine the absence of a linear relationship between two variables. Testing the linearity assumption is done with the help of Software R. One method for testing the linearity of the model is the Ramsey RESET Test (RRT). The following are the steps in the RESET test procedure.

1. Determine the restricted equation and calculate the coefficient of determination.

Restricted Model:

$$
\begin{aligned}
& Y_{i}=\beta_{0}+\beta_{1} X_{i}+\varepsilon_{i} \\
& \hat{Y}_{i}=\hat{\beta}_{0}+\hat{\beta}_{1} X_{i}
\end{aligned}
$$

With the coefficient of determination is as follows.

$$
R_{1}^{2}=1-\frac{\sum_{i=1}^{n}\left(Y_{i}-\hat{Y}_{i}\right)^{2}}{\sum_{i=1}^{n}\left(Y_{i}-\bar{Y}\right)^{2}}
$$

2. Determine the unrestricted equation and again calculate the coefficient of determination.

Unrestricted Model:

$$
\begin{aligned}
& Y_{1}^{*}=\beta_{0}^{*}+\beta_{1}^{*} X_{i}+\beta_{2}^{*} \hat{Y}_{i}^{2}+\beta_{3}^{*} \hat{Y}_{i}^{3}+\varepsilon_{i} \\
& \hat{Y}_{1}^{*}=\hat{\beta}_{0}^{*}+\hat{\beta}_{1}^{*} X_{i}+\hat{\beta}_{2}^{*} \hat{Y}_{i}^{2}+\hat{\beta}_{3}^{*} \hat{Y}_{i}^{3}
\end{aligned}
$$

With the coefficient of determination is as follows.

$$
R_{2}^{2}=1-\frac{\sum_{i=1}^{n}\left(Y_{i}-\hat{Y}_{i}^{*}\right)^{2}}{\sum_{i=1}^{n}\left(Y_{i}-\bar{Y}\right)^{2}}
$$

3. Test the linearity relationship of the model with the following hypothesis and test statistics. Hypothesis:

$\mathrm{H}_{0}: \beta_{2}=\beta_{3}=0, v s$

$\mathrm{H}_{1}$ : there is at least one $\beta_{j} \neq 0$ with $j=2,3$

Test Statistics:

$$
F=\frac{\left(R_{2}^{2}-R_{1}^{2}\right) / 2}{\left(1-R_{2}^{2}\right) /(n-2)} F_{(2, \mathrm{n}-2)}
$$


If the test statistics $F<$ tipping point $F_{(2, \mathrm{n}-2)}$ then $\mathrm{H}_{0}$ accepted, which means that the relationship between variables is linear. Otherwise if the test statistics $F>$ critical point $F_{(2, \mathrm{n}-2)}$ then $\mathrm{H}_{0}$ rejected, which means that the relationship between variables is not linear.

If the results show that the relationship between variables is not linear, then the test is continued using the Modified RRT. This test is carried out in stages starting from testing the quadratic, cubic, quartic, and quantic models.

\section{a. Quadratic Model Testing}

1. Determine the restricted model and calculate the coefficient of determination.

Restricted Model:

$$
\begin{aligned}
& Y_{i}=\beta_{0}+\beta_{1} X_{i}+\beta_{2} X_{i}^{2}+\varepsilon_{i} \\
& \hat{Y}_{i}=\hat{\beta}_{0}+\hat{\beta}_{1} X_{i}+\hat{\beta}_{2} X_{i}^{2}
\end{aligned}
$$

With the coefficient of determination is

$$
R_{1}^{2}=1-\frac{\sum_{i=1}^{n}\left(Y_{i}-\hat{Y}_{i}\right)^{2}}{\sum_{i=1}^{n}\left(Y_{i}-\bar{Y}\right)^{2}}
$$

2. Determine the unrestricted model and calculate the coefficient of determination.

Unrestricted Model:

$$
\begin{aligned}
& Y_{1}^{*}=\beta_{0}^{*}+\beta_{1}^{*} X_{i}+\beta_{2}^{*} X_{i}^{2}+\beta_{3}^{*} \hat{Y}_{i}^{2}+\beta_{4}^{*} \hat{Y}_{i}^{3}+\varepsilon_{i} \\
& Y_{1}^{*}=\hat{\beta}_{0}^{*}+\hat{\beta}_{1}^{*} X_{i}+\hat{\beta}_{2}^{*} X_{i}^{2}+\hat{\beta}_{3}^{*} \hat{Y}_{i}^{2}+\hat{\beta}_{4}^{*} \hat{Y}_{i}^{3}
\end{aligned}
$$

With the coefficient of determination is

$$
R_{2}^{2}=1-\frac{\sum_{i=1}^{n}\left(Y_{i}-\hat{Y}_{i}^{*}\right)^{2}}{\sum_{i=1}^{n}\left(Y_{i}-\bar{Y}\right)^{2}}
$$

3. Testing the quadratic model relationship with the hypothesis and test statistics as follows.

Hypothesis:

$\mathrm{H}_{0}: \beta_{3}=\beta_{4}=0, v s$

$\mathrm{H}_{1}$ : there is at least one $\beta_{j} \neq 0$ with $j=3,4$

Test Statistics:

$$
F=\frac{\left(R_{2}^{2}-R_{1}^{2}\right) / 2}{\left(1-R_{2}^{2}\right) /(n-3)} \sim F_{(2, \mathrm{n}-3)}
$$

If the test statistics $F<$ tipping point $F_{(2, \mathrm{n}-3)}$ then $\mathrm{H}_{0}$ accepted, which means that the relationship between variables is quadratic. Otherwise if the test statistics $F>$ critical point
$F_{(2, \mathrm{n}-3)}$ then $\mathrm{H}_{0}$ rejected, which means that the relationship between variables is more than quadratic.

\section{b. Cubic Model Testing}

1. Determine the restricted model and calculate the coefficient of determination.

Restricted Model:

$$
\begin{aligned}
& Y_{i}=\beta_{0}+\beta_{1} X_{i}+\beta_{2} X_{i}^{2}+\beta_{3} X_{i}^{3}+\varepsilon_{i} \\
& \hat{Y}_{i}=\hat{\beta}_{0}+\hat{\beta}_{1} X_{i}+\hat{\beta}_{2} X_{i}^{2}+\hat{\beta}_{3} X_{i}^{3}
\end{aligned}
$$

With the coefficient of determination is

$$
R_{1}^{2}=1-\frac{\sum_{i=1}^{n}\left(Y_{i}-\hat{Y}_{i}\right)^{2}}{\sum_{i=1}^{n}\left(Y_{i}-\bar{Y}\right)^{2}}
$$

2. Determine the unrestricted model and calculate the coefficient of determination.

Unrestricted Model:

$$
\begin{aligned}
& Y_{1}^{*}=\beta_{0}^{*}+\beta_{1}^{*} X_{i}+\beta_{2}^{*} X_{i}^{2}+\beta_{3}^{*} X_{i}^{3}+\beta_{4}^{*} \hat{Y}_{i}^{2}+\beta_{5}^{*} \hat{Y}_{i}^{3}+\varepsilon_{i} \\
& Y_{1}^{*}=\hat{\beta}_{0}^{*}+\hat{\beta}_{1}^{*} X_{i}+\hat{\beta}_{2}^{*} X_{i}^{2}+\hat{\beta}_{3}^{*} X_{i}^{3}+\hat{\beta}_{4}^{*} \hat{Y}_{i}^{2}+\hat{\beta}_{5}^{*} \hat{Y}_{i}^{3}
\end{aligned}
$$

With the coefficient of determination is

$$
R_{2}^{2}=1-\frac{\sum_{i=1}^{n}\left(Y_{i}-\hat{Y}_{i}^{*}\right)^{2}}{\sum_{i=1}^{n}\left(Y_{i}-\bar{Y}\right)^{2}}
$$

3. Testing the relationship of the cubic model with the hypothesis and test statistics as follows. Hypothesis:

$\mathrm{H}_{0}: \beta_{4}=\beta_{5}=0, v s$

$\mathrm{H}_{1}$ :there is at least one $\beta_{j} \neq 0$ with $j=4,5$

Test Statistics:

$$
F=\frac{\left(R_{2}^{2}-R_{1}^{2}\right) / 2}{\left(1-R_{2}^{2}\right) /(n-4)} \sim F_{(2, \mathrm{n}-5)}
$$

If the test statistics $F$ <tipping point $F_{(2, \mathrm{n}-4)}$ then $\mathrm{H}_{0}$ accepted, which means that the relationship between variables is cubic. Otherwise if the test statistics $F>$ critical point $F_{(2, \mathrm{n}-4)}$ then $\mathrm{H}_{0}$ rejected, which means that the relationship between variables is more than cubic. 


\section{c. Quartic Model Testing}

1. Determine the restricted model and calculate the coefficient of determination.

Restricted Model:

$$
\begin{aligned}
& Y_{i}=\beta_{0}+\beta_{1} X_{i}+\beta_{2} X_{i}^{2}+\beta_{3} X_{i}^{3}+\beta_{4} X_{i}^{4}+\varepsilon_{i} \\
& \hat{Y}_{i}=\hat{\beta}_{0}+\hat{\beta}_{1} X_{i}+\hat{\beta}_{2} X_{i}^{2}+\hat{\beta}_{3} X_{i}^{3}+\hat{\beta}_{4} X_{i}^{4}
\end{aligned}
$$

With the coefficient of determination is

$$
R_{1}^{2}=1-\frac{\sum_{i=1}^{n}\left(Y_{i}-\hat{Y}_{i}\right)^{2}}{\sum_{i=1}^{n}\left(Y_{i}-\bar{Y}\right)^{2}}
$$

2. Determine the unrestricted model and calculate the coefficient of determination.

Unrestricted Model:

$Y_{i}^{*}=\beta_{0}^{*}+\beta_{1}^{*} X_{i}+\beta_{2}^{*} X_{i}^{2}+\beta_{3}^{*} X_{i}^{3}+\beta_{4}^{*} X_{i}^{4}+\beta_{5} \hat{Y}_{2}^{2}+\beta_{6} \hat{Y}_{2}^{3}+\varepsilon_{i}$ ith the coefficient of determination is

$$
\begin{aligned}
& \hat{Y}_{i}^{*}=\hat{\beta}_{0}^{*}+\hat{\beta}_{1}^{*} X_{i}+\hat{\beta}_{2}^{*} X_{i} \\
& \text { With the coefficient of d } \\
& R_{2}^{2}=1-\frac{\sum_{i=1}^{n}\left(Y_{i}-\hat{Y}_{i}^{*}\right)^{2}}{\sum_{i=1}^{n}\left(Y_{i}-\bar{Y}\right)^{2}}
\end{aligned}
$$

3. Test the relationship of the quartic model with the following hypothesis and test statistics.

$\mathrm{H}_{0}: \beta_{5}=\beta_{6}=0, v s$

$\mathrm{H}_{1}$ : there is at least one $\beta_{j} \neq 0$ with $j=5,6$

Test Statistics:

$$
F=\frac{\left(R_{2}^{2}-R_{1}^{2}\right) / 2}{\left(1-R_{2}^{2}\right) /(n-5)} F_{(2, \mathrm{n}-5)}
$$

If the test statistics $F<$ tipping point $F_{(2, \mathrm{n}-5)}$ then $\mathrm{H}_{0}$ accepted, which means that the relationship between variables is quartic. Otherwise if the test statistics $F>$ critical point $F_{(2, \mathrm{n}-5)}$ then $\mathrm{H}_{0}$ rejected, which means that the relationship between variables is more than quartic.

\section{d. Quartic Model Testing}

1. Determine the restricted model and calculate the coefficient of determination.

Restricted Model:

$Y_{i}=\beta_{0}+\beta_{1} X_{i}+\beta_{2} X_{i}^{2}+\beta_{3} X_{i}^{3}+\beta_{4} X_{i}^{4}+\beta_{5} X_{i}^{5}+\varepsilon_{i}$

$R_{2}^{2}=1-\frac{\sum_{i=1}^{n}\left(Y_{i}-\hat{Y}_{i}^{*}\right)^{2}}{\sum_{i=1}^{n}\left(Y_{i}-\bar{Y}\right)^{2}}$

5. Testing the quantitative model relationship with the hypothesis and test statistics as follows.

$\mathrm{H}_{0}: \beta_{5}=\beta_{6}=0, v s$

$\mathrm{H}_{1}$ :there is at least one $\beta_{j} \neq 0$ with $j=5,6$

Test Statistics:

$$
F=\frac{\left(R_{2}^{2}-R_{1}^{2}\right) / 2}{\left(1-R_{2}^{2}\right) /(n-5)} F_{(2, \mathrm{n}-5)}
$$

If the test statistics $F$ <tipping point $F_{(2, \mathrm{n}-6)}$ then $\mathrm{H}_{0}$ accepted, which means that the relationship between the variables is quantified. Otherwise if the test statistics $F>$ critical point $F_{(2, \mathrm{n}-6)}$ then $\mathrm{H}_{0}$ rejected, which means that the relationship between the variables is more than quantitative. However, in this study, if $\mathrm{H}_{0}$ rejected, the relationship between the models is still considered quantic on the grounds of the Principle of Parsimony.

\subsection{The Estimator Properties of the SFAM Structural Model are Flexible and Robust}

Some of the estimator properties of the SFAM structural model are unbiased, efficient and consistent. The first part is estimating the theoretical nature of the model's unbias.

$$
\hat{Y}_{i}=\hat{\beta}_{0}+\hat{\beta}_{1} X_{i}+\hat{\beta}_{2} X_{i}^{2}+\hat{\beta}_{3} X_{i}^{3}+\hat{\beta}_{4} X_{i}^{4}+\hat{\beta}_{5} X_{i}^{5}
$$

With the coefficient of determination is

$$
R_{1}^{2}=1-\frac{\sum_{i=1}^{n}\left(Y_{i}-\hat{Y}_{i}\right)^{2}}{\sum_{i=1}^{n}\left(Y_{i}-\bar{Y}\right)^{2}}
$$

4. Determine the unrestricted model and calculate the coefficient of determination.

Unrestricted Model:

$$
\begin{aligned}
& Y_{i}^{*}=\beta_{0}^{*}+\beta_{1}^{*} X_{i}+\beta_{2}^{*} X_{i}^{2}+\beta_{3}^{*} X_{i}^{3}+\beta_{4}^{*} X_{i}^{4}+\beta_{5}^{*} X_{i}^{5}+\beta_{6} \hat{Y}_{i}^{2}+\beta_{7} \hat{Y}_{i}^{3}+\varepsilon_{i} \\
& \hat{Y}_{i}^{*}=\hat{\beta}_{0}^{*}+\hat{\beta}_{1}^{*} X_{i}+\hat{\beta}_{2}^{*} X_{i}^{2}+\hat{\beta}_{3}^{*} X_{i}^{3}+\hat{\beta}_{4}^{*} X_{i}^{4}+\hat{\beta}_{5}^{*} X_{i}^{5} \hat{\beta}_{6} \hat{Y}_{i}^{2}+\hat{\beta}_{7} \hat{Y}_{i}^{3}
\end{aligned}
$$


Predictor Properties: Unavailable (Not Biased)

$\underset{\%}{Y}=\mathbf{X} \underset{\sigma}{\beta}+\underset{\%}{\varepsilon}$

$\hat{\beta}=\left(\mathbf{X}^{\prime} \mathbf{X}\right)^{-1} \mathbf{X}^{\prime} Y_{\%}$

$\hat{\beta}=\left[\left(\mathbf{X}^{\prime} \mathbf{X}\right)^{-1} \mathbf{X}^{\prime}\right]\left[\mathbf{X} \beta+\boldsymbol{\sigma}_{\%}\right]$

$\hat{\beta}=\left(\mathbf{X}^{\prime} \mathbf{X}\right)^{-1} \mathbf{X}^{\prime} \mathbf{X} \beta+\left(\mathbf{X}^{\prime} \mathbf{X}\right)^{-1} \mathbf{X}_{\%}^{\prime} \varepsilon$

$\hat{\beta}=\beta+\left(\mathbf{X}^{\prime} \mathbf{X}\right)^{-1} \mathbf{X}^{\prime} \varepsilon_{\%}$

$E(\hat{\beta})=E(\underset{\%}{\beta})+E\left(\left(\mathbf{X}^{\prime} \mathbf{X}\right)^{-1} \mathbf{X}_{\%}^{\prime} \varepsilon_{\%}\right)$

$E(\hat{\beta})=\underset{\text { On }}{\beta}+\left(\mathbf{X}^{\prime} \mathbf{X}\right)^{-1} \mathbf{X}^{\prime} E\left(\varepsilon_{\%}^{\varepsilon}\right)$

$E(\hat{\beta})=\beta$

From the results of the unbiased prediction properties:

\section{Lemma 2.1 which has a shape}

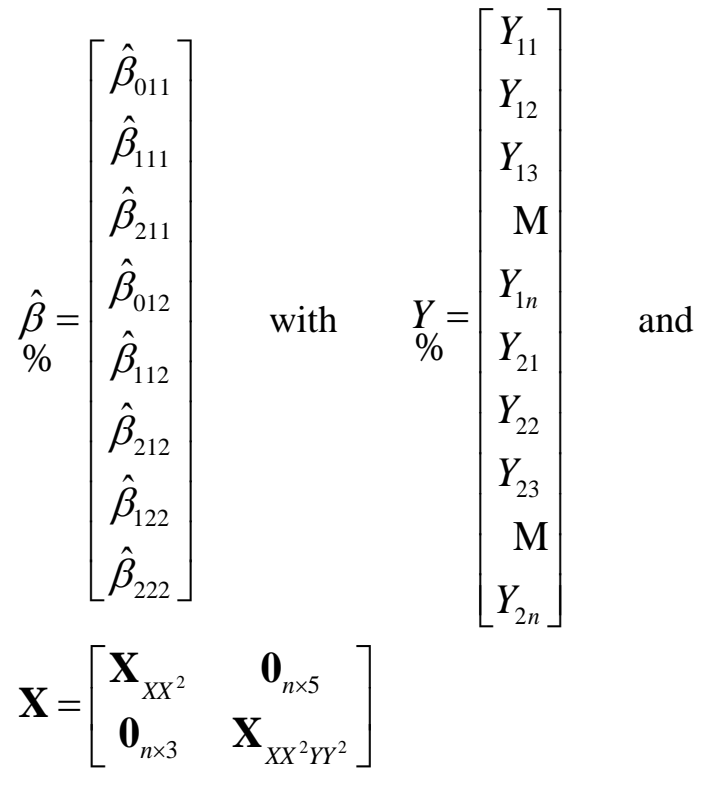

\section{Lemma 2.2 which has a shape}

$\hat{\beta}=\left[\begin{array}{c}\hat{\beta}_{1} \\ \% \\ \hat{\beta}_{2} \\ \% \\ \hat{\beta}_{3} \\ \% \\ \mathrm{M} \\ \hat{\beta}_{q} \\ \sigma_{2}\end{array}\right]$

with $\quad Y=\left[\begin{array}{c}Y \\ \% \\ Y \\ \% \\ Y \\ \% \\ \mathrm{M} \\ Y \\ \% \\ \%\end{array}\right]$

and

Lemma 2.3 which has a shape

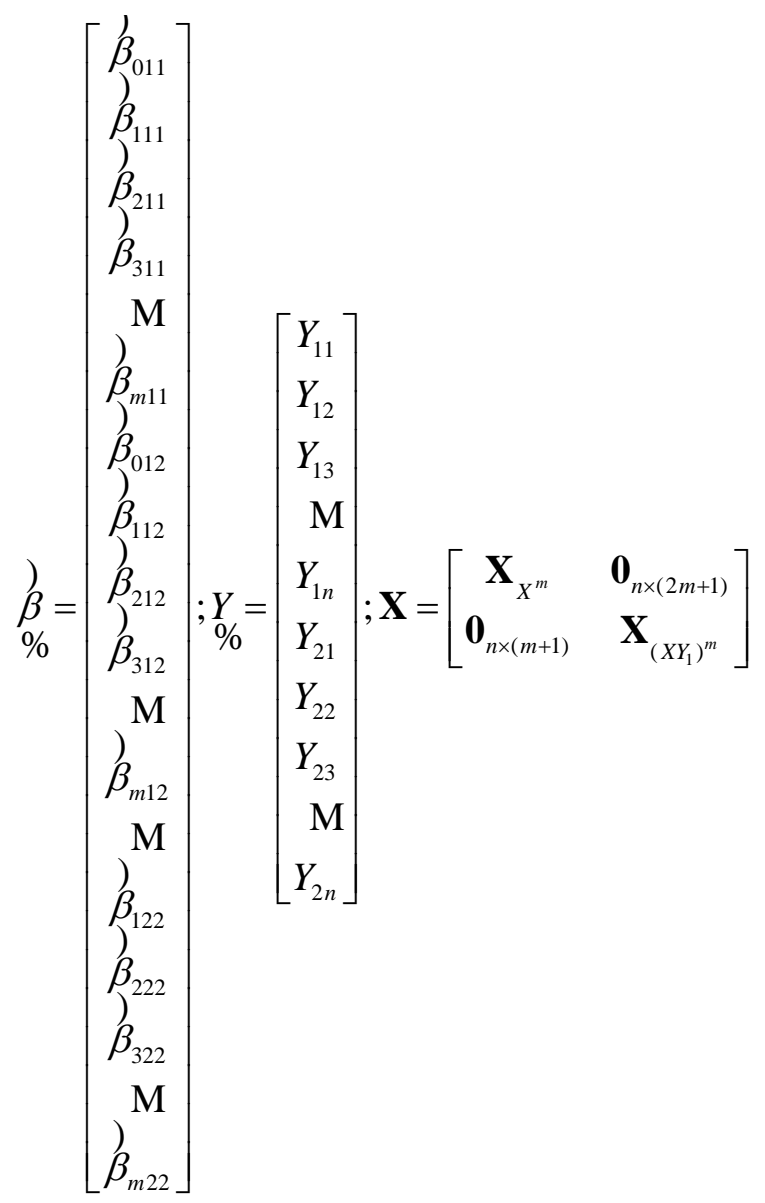




\section{Lemma 2.4 which has a shape}

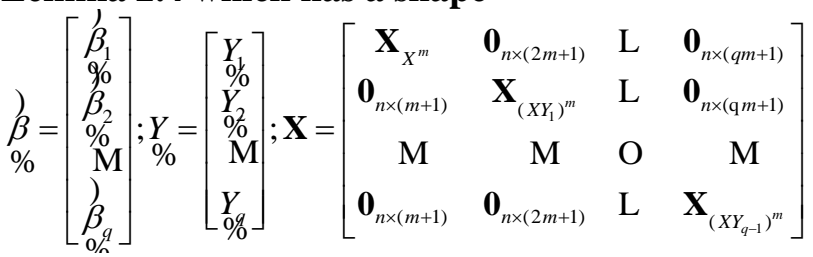

\section{Predictor Nature: Efficient}

The resampling method comparison is measured based on the relative efficiency value. Relative efficiency is calculated by comparing the variance between two parameter estimators. The relative efficiency of the two estimators can be written as follows.

$$
\operatorname{eff}\left(\hat{\beta}_{J K}, \hat{\beta}_{B S}\right)=\frac{V\left(\hat{\beta}_{J K}\right)}{V\left(\hat{\beta}_{J K}\right)}
$$

Information:

$$
\text { eff }\left(\beta_{B S}, \beta_{J K}\right)
$$

$V\left(\hat{\beta}_{J K}\right)$

$V\left(\hat{\beta}_{B S}\right)$

If the result of the calculation of efficiency is more than 1 , estimator $\beta_{B S}$ more efficient than estimator $\beta_{J K}$. Conversely, if the calculated efficiency is less than 1 , estimator $\beta_{J K}$ more efficient than estimator $\beta_{B S}$. If the result of the calculation of efficiency is equal to 1 , the two estimators are equally efficient.

\section{Predictor Nature: Consistent}

Estimator consistency in resampling can be indicated by the bias value, namely the difference (distance) between the estimator and the parameter. The following is a formula for calculating the estimator bias in resampling for the relationship between exogenous variables $X$ and endogenous $Y_{1}$.

$$
\operatorname{bias}_{F}=\operatorname{bias}_{F}\left(\hat{\beta}_{X Y_{1}}, \beta_{X Y_{1}}\right)=\left|E_{F}[s(x)]-t(F)\right|
$$

Where:

bias $_{F}$ : bias value

$\hat{\beta}_{X Y_{1}}$ : parameter estimator of the process resampling

$\beta_{X Y_{1}}:$ parameter

$s(x)$ : parameter estimator of the process resampling $t(F):$ parameter
Good resampling results will follow a simulation approach that is based on averages.

$\hat{\beta}^{*}()=.\sum_{b=1}^{B} \frac{\hat{\beta}^{*}(b)}{B}=\sum_{b=1}^{B} \frac{\mathrm{s}\left(\mathrm{x}_{b}^{*}\right)}{B}$

Where $\hat{\beta}^{*}($.) is the average parameter estimator obtained from the resampling process. Thus, the bias is based on replicas $B$ is to replace $E_{F}[s(x)]$ with $\hat{\beta}^{*}($.).

bias $_{F}=\left|\hat{\beta}^{*}()-.t(F)\right|$

Biased value $\left(\right.$ bias $\left._{F}\right)$ can be used to determine the consistency of estimators obtained from the sample.

\section{Data Application}

\subsection{Hypothesis Testing of Flexible and Sturdy SFAM Structural Models}

The following section tests the structural model hypotheses with 17 primary data. The application

ariowassestriater

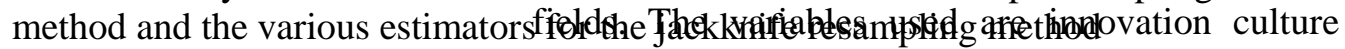

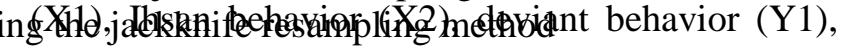
and employee performance (Y2). The research unit

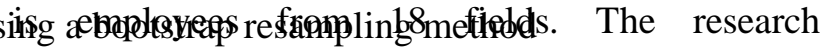
instrument used was a questionnaire. The feasibility test of the research instrument was carried out by testing the validity and reliability testing.

Validity and reliability tests were carried out on the test data. The research instrument is said to be valid if the corrected item-total correlation value is positive $\geq 0.3$, while the research instrument is said to be reliable when the Cronbach Alpha reliability coefficient is $\geq 0.6$. In this study, the trial involved 50 employees. The results of checking the validity and reliability of the research instruments are listed

\begin{tabular}{|c|c|c|c|c|}
\hline Variable & Indicator & Valid Items & $\begin{array}{l}\text { Invali } \\
\text { d Item }\end{array}$ & $\begin{array}{l}\text { Cronbach' } \\
\text { s Alpha }\end{array}$ \\
\hline \multirow{2}{*}{$\begin{array}{l}\text { Innovation } \\
\text { Culture } \\
\text { (X1) }\end{array}$} & Habit (X11) & $1,2,3,4,5,6$ & - & \multirow[b]{2}{*}{0.781} \\
\hline & $\begin{array}{l}\text { Viewpoint } \\
\text { (X12) }\end{array}$ & $1,2,3,4,5,6,7,8$ & - & \\
\hline \multirow[t]{4}{*}{$\begin{array}{l}\text { Ihsan's } \\
\text { Behavior } \\
\text { (X2) }\end{array}$} & $\begin{array}{l}\text { Does the job } \\
\text { perfect } \\
(\mathrm{X} 21)\end{array}$ & $1,2,3,4$ & - & \multirow{4}{*}{0.821} \\
\hline & $\begin{array}{l}\text { Repay more } \\
\text { kindness } \\
(\mathrm{X} 22)\end{array}$ & $1,2,3$ & - & \\
\hline & $\begin{array}{l}\text { Reduce as } \\
\text { optimal as } \\
\text { possible the } \\
\text { unpleasant } \\
\text { consequence } \\
\mathrm{s}(\mathrm{X} 23)\end{array}$ & $1,2,3$ & - & \\
\hline & $\begin{array}{ll}\begin{array}{l}\text { As } \\
\text { solution }\end{array} & \text { a } \\
\end{array}$ & $1,2,3$ & - & \\
\hline
\end{tabular}
in Table 1.

Table 1 . Validity and reliability examination results 


\begin{tabular}{|c|c|c|c|c|}
\hline Variable & Indicator & Valid Items & $\begin{array}{l}\text { Invali } \\
\text { d Item }\end{array}$ & $\begin{array}{l}\text { Cronbach' } \\
\text { s Alpha }\end{array}$ \\
\hline & $\begin{array}{l}\text { when } \\
\text { optimal } \\
\text { justice } \\
\text { cannot be } \\
\text { realized } \\
\text { (X24) }\end{array}$ & & & \\
\hline & $\begin{array}{l}\text { As a logical } \\
\text { consequence } \\
\text { of faith } \\
\text { (X25) }\end{array}$ & $1,2,3$ & - & \\
\hline & $\begin{array}{l}\text { As an } \\
\text { investment } \\
\text { in future } \\
\text { success } \\
\text { (X26) }\end{array}$ & $1,2,3$ & - & \\
\hline \multirow{2}{*}{$\begin{array}{l}\text { Deviant } \\
\text { Behavior } \\
\text { (Y1) }\end{array}$} & $\begin{array}{l}\text { Interpersona } \\
1 \text { drift (Y11) }\end{array}$ & $1,2,3,4,5,6$ & - & \multirow[b]{2}{*}{0.780} \\
\hline & $\begin{array}{l}\text { Deviations } \\
\text { to the } \\
\text { organization } \\
\text { (Y12) }\end{array}$ & $\begin{array}{l}1,2,2,4,5,6,7,8 \\
9\end{array}$ & - & \\
\hline \multirow[t]{6}{*}{$\begin{array}{l}\text { Employee } \\
\text { Performanc } \\
\text { e (Y2) }\end{array}$} & $\begin{array}{l}\text { Quality } \\
\text { quality of } \\
\text { work (Y21) }\end{array}$ & $1,2,3,4,5,6$ & - & \multirow{6}{*}{0.853} \\
\hline & $\begin{array}{l}\text { Power and } \\
\text { cost } \\
\text { efficiency } \\
\text { (Y22) }\end{array}$ & $1,2,3,4$ & - & \\
\hline & $\begin{array}{l}\text { Initiative } \\
\text { (Y23) }\end{array}$ & $1,2,3,4,5$ & - & \\
\hline & $\begin{array}{l}\text { Mastery of } \\
\text { duty (Y24) }\end{array}$ & $1,2,3,4,5$ & - & \\
\hline & $\begin{array}{l}\text { Reliability } \\
\text { (Y25) }\end{array}$ & $1,2,3$ & - & \\
\hline & $\begin{array}{l}\text { Attendance } \\
\text { at } \quad \text { work } \\
\text { (Y26) }\end{array}$ & $1,2,3,4,5$ & - & \\
\hline
\end{tabular}

From the results of the validity and reliability test, it shows that all items are valid and reliable. so it is necessary to evaluate the research instrument. Furthermore, data collection was carried out in 18 fields and applied path analysis to the data obtained. One application of the analysis included in this paper is from PT Bank Rakyat Indonesia (Persero) Tbk

1) Linearity Test

The test that can be used to determine whether the variables have a linear relationship or not is the Ramsey RESET Test. The results of linearity testing between variables are listed in Table 2.

Table 2. Test Results of Linearity Assumptions

\begin{tabular}{|l|l|l|}
\hline Variable & P-value & Result \\
\hline X1 with Y1 & 0.003 & Non Linear \\
\hline X2 with Y1 & 0,000 & Non Linear \\
\hline X1 with Y2 & 0.049 & Non Linear \\
\hline X2 with Y2 & 0.931 & Linear \\
\hline Y1 to Y2 & 0.948 & Linear \\
\hline
\end{tabular}

Based on Table 2, it can be seen that there are three non-linear relationships. So, the test was continued by performing the Modified Ramsey RESET Test so that the results in Table 3 were produced.

Table 3. Test Results of Modified Ramsey RESET Test

\begin{tabular}{|l|l|}
\hline Variable & Result \\
\hline X1 with Y1 & Quadratic \\
\hline X2 with Y1 & Quadratic \\
\hline X1 with Y2 & Quadratic \\
\hline
\end{tabular}

The result of parameter estimation can be seen in Figure 2.

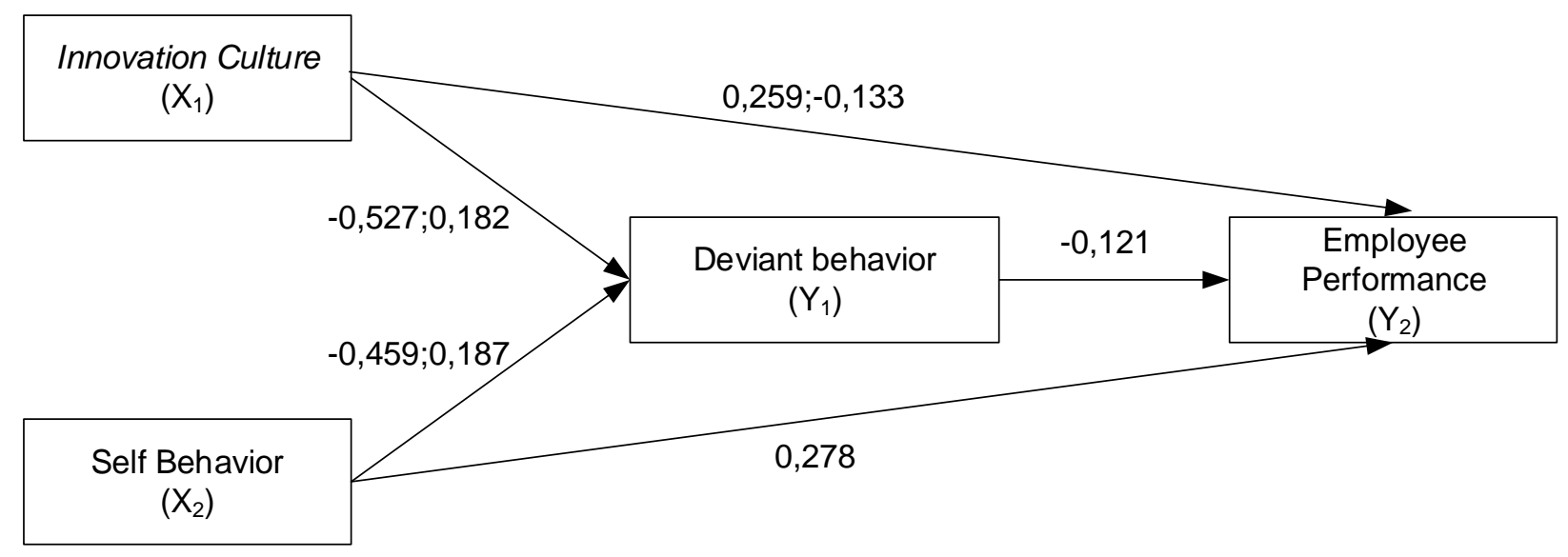

Fig. 2: Path diagram of PT Bank Republik Indonesia (Persero) Tbk

The path analysis model for data from Bank Republik Indonesia (BRI) can be written as follows:

$$
Z_{Y_{1}}=-0,527 Z_{X_{1}}+0,182 Z_{X_{1}}^{2}-0,459 Z_{X_{2}}+0,187 Z_{X_{2}}^{2}
$$




$$
Z_{Y_{2}}=0,259 Z_{X_{1}}-0,133 Z_{X_{1}}^{2}+0,278 Z_{X_{2}}-0,121 Z_{Y_{1}}
$$

the normal distribution, so that a histogram is formed that forms a normal curve.

Hypothesis testing is done by resampling Bootstrap. Figure 3 shows that by retrieval of the sample 1000 times, the data estimator approaches
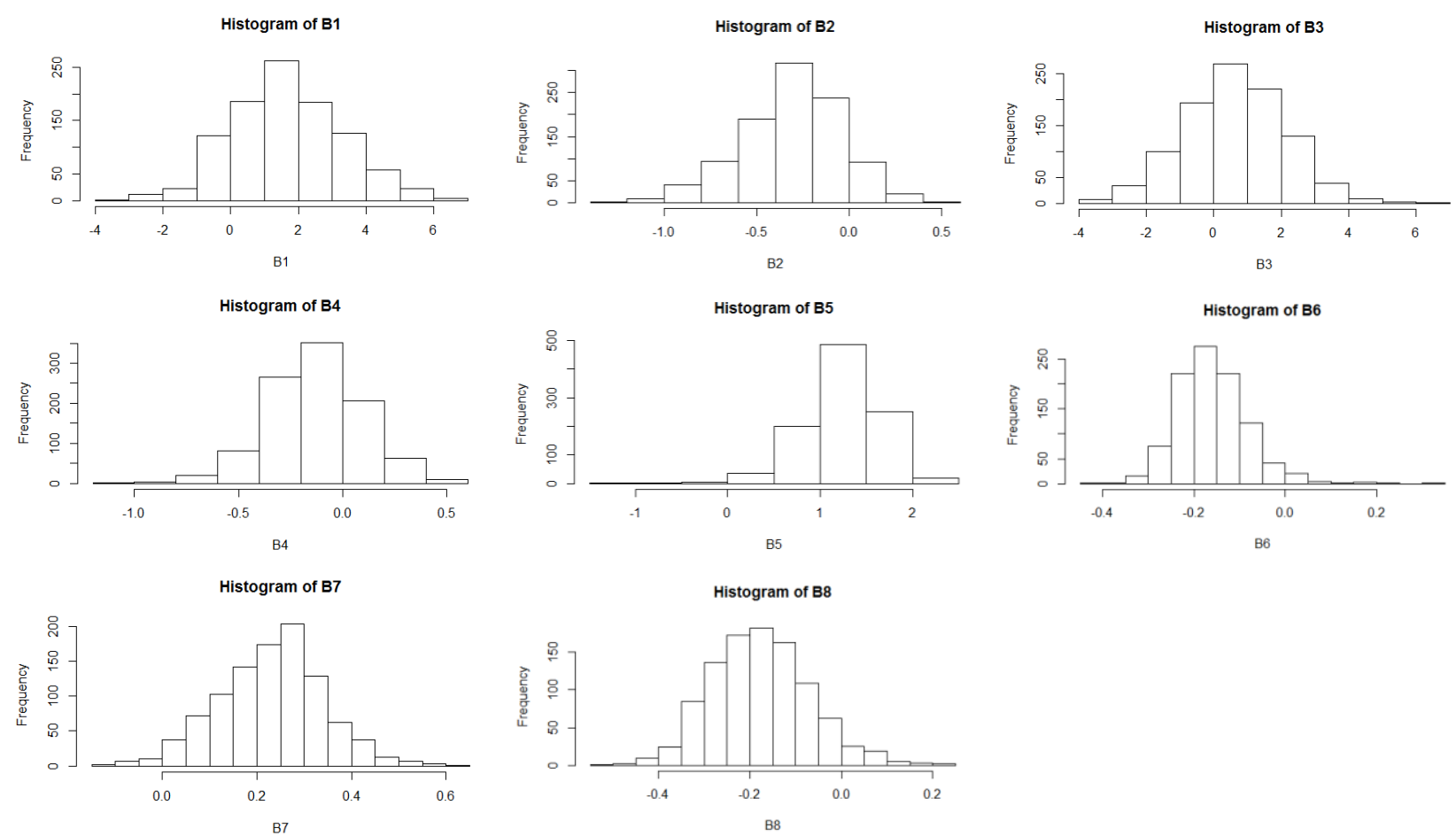

Fig. 3: Parameter Estimator Histogram with Bootstrap

The results of testing the direct effect hypothesis are shown in Table 4.

Table 4. Hypothesis Testing Results Direct Effect

\begin{tabular}{|l|l|l|l|l|}
\hline Variable & $\begin{array}{l}\text { Path } \\
\text { Coefficient } \\
(\text { OLS) }\end{array}$ & $\begin{array}{l}\text { Standard } \\
\text { Error } \\
\text { (Bootstrap) }\end{array}$ & P-value & Result \\
\hline $\mathrm{X}_{1 \rightarrow \mathrm{Y}_{1}}$ & -0.527 & 0.220 & 0,000 & Significant \\
\hline $\mathrm{X}_{1}^{2} \rightarrow \mathrm{Y}_{1}$ & 0.182 & 0.036 & 0,000 & Significant \\
\hline $\mathrm{X}_{2} \rightarrow \mathrm{Y}_{1}$ & -0.459 & 0.202 & 0.004 & Significant \\
\hline $\mathrm{X}_{2}^{2} \rightarrow \mathrm{Y}_{1}$ & 0.187 & 0.030 & 0,000 & Significant \\
\hline $\mathrm{X}_{1} \rightarrow \mathrm{Y}_{2}$ & 0.259 & 0.058 & 0,000 & Significant \\
\hline $\mathrm{X}_{1}^{2} \rightarrow \mathrm{Y}_{2}$ & -0.133 & 0.011 & 0,000 & Significant \\
\hline $\mathrm{X}_{2} \rightarrow \mathrm{Y}_{2}$ & 0.278 & 0.016 & 0,000 & Significant \\
\hline $\mathrm{Y}_{1} \rightarrow \mathrm{Y}_{2}$ & $-0,121$ & 0.015 & 0,000 & Significant \\
\hline
\end{tabular}

Based on Table 4, it can be seen that the $\mathrm{p}$ value $<\alpha(0.05)$ which results in the rejection of $\mathrm{H} 0$ and it can be concluded that there is a significant effect on this pathway. The second equation can be interpreted that every increase of one unit of Ihsan behavior will increase employee performance by 
0.278 units and every increase of one unit of deviant behavior will decrease employee performance by 0.121 units assuming other variables remain.

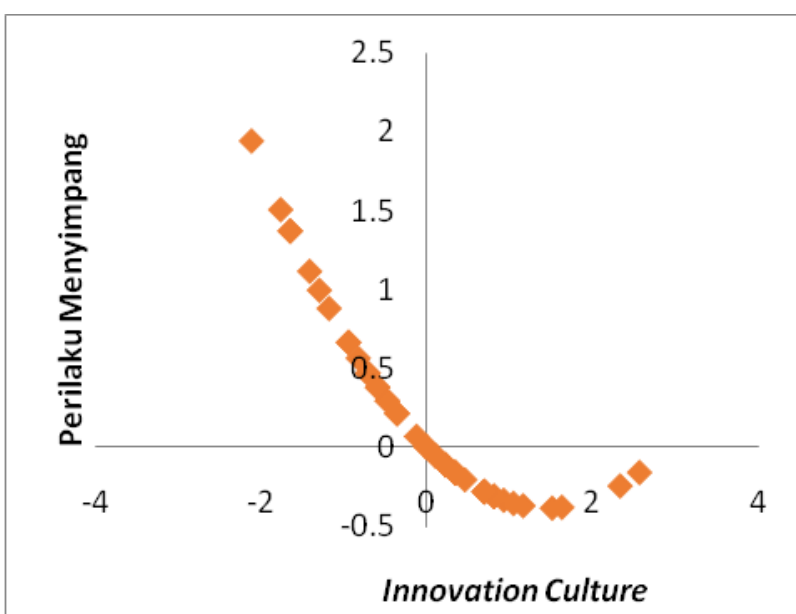

Fig. 4: The Curve of the Relationship between Innovation Culture and Deviant Behavior

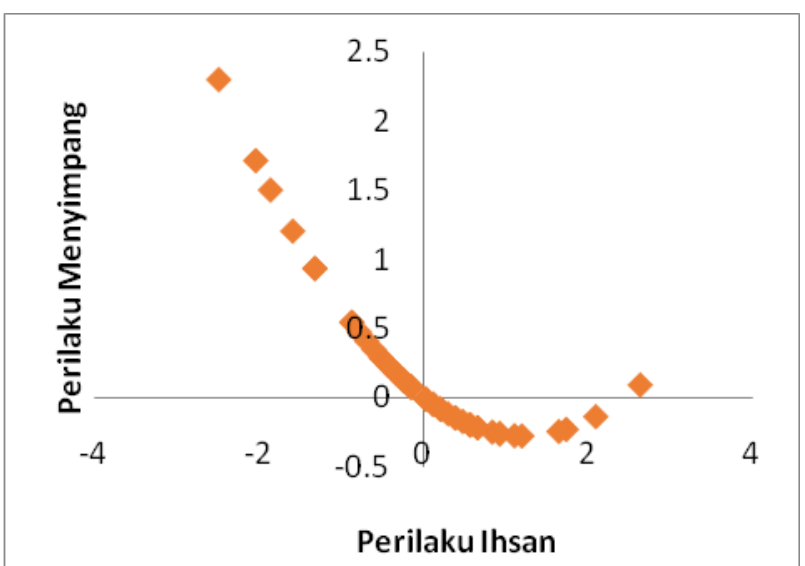

Fig. 5: The Curve of Relationship between Ihsan Behavior and Deviant Behavior

From the first equation, Figure 3 shows that the high innovation culture will reduce the employee's desire to behave defiantly. However, at the optimum point of -0.381 , the desire to behave deviantly can increase by assuming that the other variables are constant. Figure 4 also shows that the higher the Ihsan behavior, the lower the employee's desire to behave defiantly. At the optimum point of -0.281 , the employee's desire to commit deviant actions will increase along with the high Ihsan behavior assuming other variables are constant.

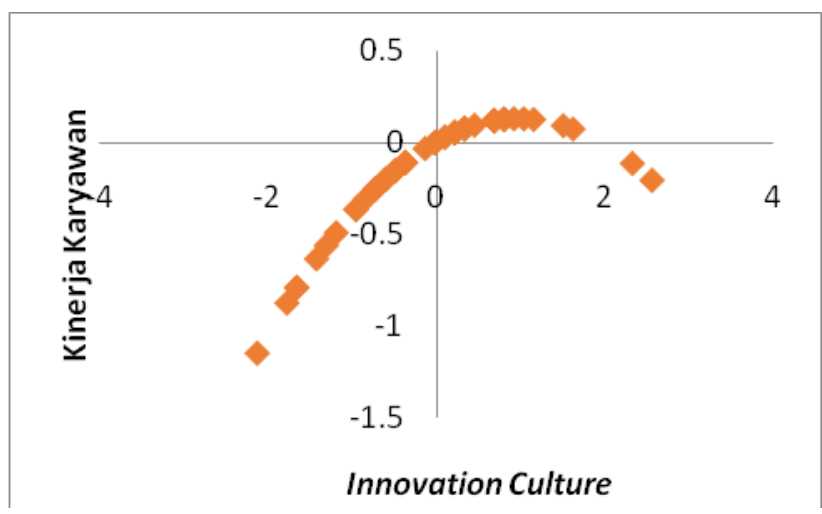

Fig. 6: The Curve of the Relationship between Innovation Culture and Employee Performance

From the second equation, Figure 5 shows that the high innovation culture will improve the performance of BRI employees. However, innovation culture can reduce employee performance if it passes the optimum point of 0.126 assuming other variables remain. The results of the path analysis in this study indicate that the amount of data diversity described by the model is $44.5 \%$, while the remaining $55.5 \%$ is explained by other variables not included in the model.

\section{Conclusion}

Based on the results of the study, it can be concluded that there is a significant influence between innovation culture and self behavior on deviants. In addition, there is also a significant influence between innovation culture, self behavior, and deviant behavior on employee performance.

The conclusions are get the development of a flexible structural model of the relationship between variables. Among them, lemma has been produced to form flexible models in the form of simple structural relationships involving single exogenous, single intervening endogenous, and single pure endogenous; a theorem has been generated for the estimation of flexible model parameters / functions in the form of simple structural relationships involving single exogenous, single intervening endogenous, and single pure endogenous; lemma has been produced to form flexible models in the form of complex structural relationships involving exogenous $\mathrm{p}$, intervening endogenous $\mathrm{m}$, and pure endogenous q; Theorem has been generated for estimating parameters / functions of flexible models in the form of complex structural relationships involving exogenous $\mathrm{p}$, intervening endogenous $\mathrm{m}$.

Obtain a structural model that is robust with the assumptions of normality and homoscedasticity. Among them is the simple structural model theorem 
of efficient and consistent resampling approach; has produced complex structural model theorem with efficient and consistent resampling approach; has produced a simple structural model theorem with a weighted approach to solving the nonheteroscedasticity case; has produced the theorem of a complex structural model with a weighted approach to solving the non-heteroscedasticity case.

Obtain estimator properties from the flexible and robust SFAM structural model. Among them are the theorems of investigating the unbiased nature of simple structural models; have generated theorems investigating the unbiased nature of complex structural models; investigated the asymptotic nature of the simple structural model function obtained; has investigated the asymptotic nature of the function of the complex structural model obtained.

Obtaining hypothesis testing of each relationship built from the flexible and robust SFAM structural model. From the results of hypothesis testing, it was concluded that of the 18 institutions studied, seven agencies had a non-linear model, while 11 other agencies had a linear model.

Further research can use the SFAM structural model of this study according to the case to be tested. The SFAM model formed in this study has the advantage of being flexible and strong, because it does not pay attention to assumptions and produces unbiased results.

\section{Acknowledgements:}

We are very grateful to experts for their appropriate and constructive suggestions to improve this paper.

\section{References:}

[1] Asrun. (2014). Kepemimpinan Spiritual: Pengaruhnya Terhadap Spiritualitas di Tempat Kerja, Kepuasan Kerja, dan Perilaku Menyimpang di Tempat Kerja (Disertasi, Universitas Brawijaya).

[2] Chen, H., and Wang, Y. (2011). A Penalized Spline Approach to Functional Mixed Effects Model Analysis. Biometrics, 67(1), 861-870

[3] Chin, W. Wynne. 2006. Overview of the PLS Method.

[4] Dillon, W. R. \& Goldstein, M. 1984. Multivariate Analysis Methods and Applicationds. New York: John Wiley dan Sons

[5] Garson, G. D. (2013). Path analysis. Asheboro: Statistical Associates Publishing.
[6] Hair, Jr., J.F., R.E Anderson, R.L. Tatham and W.C. Black. 2010. Multivariate Data Analysis with Reading. Macmillan Pub. Company. New York.

[7] Handayanto. (2014). Pengaruh Budaya Organisasi Terhadap Kepemimpinan, Nilai Personal, dan Perilaku Ihsan di Rumah Sakit Islam Masyitoh Bangil (Disertasi, Universitas Brawijaya).

[8] Heckman, N., Lockhart R., and Nielsen J.D. (2009). Penalized Regression, Mixed Effects Models and Appropriate Modelling. Retrieved, December, 12, 2012. Website: http://www.stat.ubc.ca/ nancy/pubs/lmetechrep ort.pdf.

[9] Joreskog, K and D. Sorbom. 1996. LISREL 8 : User's Reference Guide. Second Edition. Chicago: Scientific Software International, Inc.

[10] Solimun. 2010. Analisis Multivariat Pemodelan Struktural. Malang: Citra

[11] Van der Seijs, M. V., de Klerk, D., \& Rixen, D. J. (2016). General framework for transfer path analysis: History, theory and classification of techniques. Mechanical Systems and Signal Processing, 68, 217-244.

[12] Verbekke, G., Fiews, S., Molenberghs, G., and Davidian, M. (2014). The Analysis of Multivariate Longitudinal Data: A Review. Statistical Methods in Medical Research, 23(1), 42-59.

[13] Wiryatmojo, S., (2012). Pengaruh Kompetensi dan Motivasi Terhadap Intuisi dan Kinerja Aparat Reserse (Disertasi, Universitas Brawijaya).

[14] Iwu, C. G., Eze, F. I., Opute, A. P., Dongo, G. U., Dongo, O. W. (2021). Scavenging for Survival and its Health Implications. The Nexus between Unemployment and Ill-health, WSEAS Transactions on Environment and Development, 17, 1-18.

[15] Amirudin, A., Saputra, J., Afrizal, T., Latip, M., Tarmizi, A. (2021). Investigating the COVID-19 Self-Isolation Policy and Its Impact on Socioeconomic of Vulnerable Groups: An application of Rational and Non-Rational Thinking Models, WSEAS Transactions on Environment and Development, 17, 604-613.

[16] Kraus, K., Kraus, N., Nikiforov, P., Pochenchuk, G., Babukh, I. (2021). Information and Digital Development of Higher Education in the Conditions of Innovatyzation Economy of Ukraine, WSEAS Transactions on Environment and Development, 17, 659-671. 
Contribution of Individual Authors to the Creation of a Scientific Article (Ghostwriting Policy)

Adji Achmad Rinaldo Fernandes preparation, creation and of the published work, specifically writing the initial draft (including substantive translation). Solimun carried out conceptualization of the concept.

\section{Creative Commons Attribution License 4.0}

(Attribution 4.0 International, CC BY 4.0)

This article is published under the terms of the Creative Commons Attribution License 4.0

https://creativecommons.org/licenses/by/4.0/deed.en US 